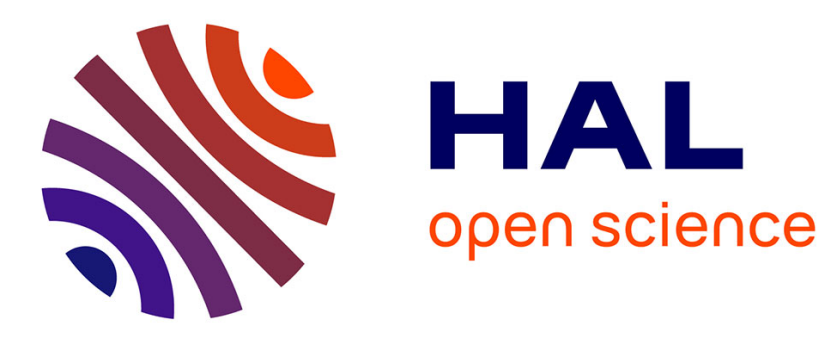

\title{
Measuring influence in command games
}

Michel Grabisch, Agnieszka Rusinowska

\section{To cite this version:}

Michel Grabisch, Agnieszka Rusinowska. Measuring influence in command games. Social Choice and

Welfare, 2009, 33 (2), pp.177-209. 10.1007/s00355-008-0350-8 . halshs-00445126

\section{HAL Id: halshs-00445126 \\ https://shs.hal.science/halshs-00445126}

Submitted on 7 Jan 2010

HAL is a multi-disciplinary open access archive for the deposit and dissemination of scientific research documents, whether they are published or not. The documents may come from teaching and research institutions in France or abroad, or from public or private research centers.
L'archive ouverte pluridisciplinaire HAL, est destinée au dépôt et à la diffusion de documents scientifiques de niveau recherche, publiés ou non, émanant des établissements d'enseignement et de recherche français ou étrangers, des laboratoires publics ou privés. 


\title{
Measuring influence in command games ${ }^{\star}$
}

\author{
MICHEL GRABISCH${ }^{1}$ and AGNIESZKA RUSINOWSKA ${ }^{2}$ \\ 1 Université Paris I Panthéon-Sorbonne \\ Centre d'Economie de la Sorbonne, 106-112 Bd de l'Hôpital, 75013 Paris, France \\ michel.grabisch@univ-paris1.fr \\ 2 GATE, CNRS UMR 5824 - Université Lumière Lyon 2 - Ecole Normale Supérieure LSH \\ 93, Chemin des Mouilles - B.P.167, 69131 Ecully Cedex, France \\ rusinowska@gate.cnrs.fr
}

\begin{abstract}
In the paper, we study a relation between command games proposed by Hu and Shapley and an influence model. We show that our framework of influence is more general than the framework of the command games. We define several influence functions which capture the command structure. These functions are compatible with the command games, in the sense that each commandable player for a coalition in the command game is a follower of the coalition under the command influence function. Some of the presented influence functions are equivalent to the command games, that is, they are compatible with the command games, and additionally each follower of a coalition under the command influence function is also a commandable player for that coalition in the command games. For some influence functions we define the equivalent command games. We show that not for all influence functions the compatible command games exist. Moreover, we propose a more general definition of the influence index and show that under some assumptions, some power indices, which can be used in the command games, coincide with some expressions of the weighted influence indices. Both the Shapley-Shubik index and the Banzhaf index are equal to a difference between the weighted influence indices under some influence functions, and the only difference between these two power indices lies in the weights for the influence indices. An example of the Confucian model of society is broadly examined.
\end{abstract}

JEL Classification: C7, D7

Keywords: influence function, follower, influence index, command game, commandable player, Shapley-Shubik index, Banzhaf index, Coleman indices, König-Bräuninger index

\section{Introduction}

Studying an interaction and an influence among players in voting situations exposes many challenging problems to be solved. In traditional framework of power indices (see e.g., $[1-6,12,15-21,23]$, see also [7] for an overview), neither interaction nor influence among the voters is assumed. The concept of the interaction or cooperation among players in a cooperative game is studied, for instance, in [8], where players in a coalition are said to exhibit a positive (negative) interaction when the worth of the coalition is greater (smaller) than the sum of the individual worths. The authors present an axiomatization of the interaction indices which are extensions of the Shapley and Banzhaf values.

Another approach is presented in [13,14], where the command structure of Shapley [22] is applied to model players' interaction relations by simple games. For each player, boss sets and approval sets are introduced. While the boss sets are defined as the 'sets of individuals that the player must obey, regardless of his own judgment or desires', the 'consent of the approval set is sufficient to allow the player to act, if he wishes' [14].

\footnotetext{
${ }^{\star}$ The authors wish to gratefully thank two anonymous referees for useful suggestions concerning this paper.
} 
Based on the boss and approval sets, a simple game called the command game for a player is built. Furthermore, an equilibrium authority distribution is formulated to which the Shapley-Shubik index is applied.

Coming still from a different direction is an approach proposed by Hoede and Bakker [11], where the authors talk explicitly about an influence between players in a social network, and they define the Hode-Bakker index. Players, who are to make a yes-no decision, have their inclinations to say either 'yes' or 'no', but due to the influence by the others, they can decide differently from their inclinations. Formally speaking, to each possible configuration $i$ of individual inclinations, the configuration $B i$ of decisions of all players is assigned, where $B$ denotes the influence function. Then, based on $B i$, a group decision either 'yes' or 'no' is made.

The model of Hoede and Bakker is the point of departure for our research on influence. As noticed in [10], despite the interest of the framework of the Hoede-Bakker index in a situation where influence exists, this index does not give a full description of the situation, in the sense that it hides the actual role of the influence function $B$. Many economic, political, real-life situations may be modeled as a social network with players influencing each other. It is therefore very important to study different ways of defining influence between agents, and to investigate useful tools that will fully measure and analyze the influence. Motivated by the importance of studying these issues in detail, we have decided to conduct a broad research on influence between players in a social network. In [10], we introduce and study weighted influence indices of a coalition on a player, and consider different influence functions. In [9] we extend the yes-no model to a multi-choice game, and assume that players have a totally ordered set of possible actions instead of just two actions 'yes' or 'no'. Each player has an inclination to choose one of the actions. Consequently, generalized influence indices are investigated.

What are the differences and advantages of the influence model in contrast to standard power indices? Roughly speaking, a power index of a player gives an answer to the question of effect the player has on the outcome in a voting situation, or differently speaking, to the question of decisiveness of that player. In a standard voting situation, once a proposal is submitted, voters cast votes, voting either 'yes' or 'no'. A vote configuration gives a possible result of voting, and the winning configurations are the ones that lead to the passage of the proposal. A player is said to be decisive if his vote coincides with the voting result, and if by changing his vote, he changes the result of voting. The framework of influence which we study is broader than voting games, where the standard power indices are defined. Our analysis starts 'one step earlier', before voting takes place. As mentioned above, in our model, voters are settled in a social network, they are inclined to make one of the possible decisions, but they may be influenced by the others, and consequently, they may vote differently from their original inclinations. One of the tools that we introduce in such a broad framework of influence, is the concept of influence index. This tool measures particularly the influence between players, i.e., the ability to make agents vote differently from their original inclinations, and as such, it is basically different from the concept of power index, which focuses on the effect that a player has on the outcome. Nevertheless, if we make some assumptions 'linking' a voting game to the influence model, standard power indices can also be expressed by our weighted influence indices. For instance, we may define a winning coalition in a voting game as a coalition of players with the same inclination such that a given agent always follows this 
coalition (assuming that all remaining players have a different inclination). This link will be precisely used in the sequel to relate command games and influence functions.

The aim of the present paper is to study the relation between the command games considered by $\mathrm{Hu}$ and Shapley [13,14], and the influence model investigated in $[9,10]$. We show that our framework of influence is more general than the framework of command games. In the influence model, many different influence functions may be proposed. Depending on how the influence function is chosen, the influence-function approach provides a much wider spectrum, compared to the command-game approach. In the present paper, we define several influence functions which capture the command structure proposed by $\mathrm{Hu}$ and Shapley. These influence functions, called command influence functions, explain the command games, by specifying how exactly players decide according to the command structure. The command influence functions that we define are compatible with the command games, in the sense that each commandable player for a coalition in the command game is a follower of the coalition under the command influence function. Some of the presented command influence functions are equivalent to the command games, that is, they are compatible with the command games, and additionally each follower of a coalition under the command influence function is also a commandable player for that coalition in the command games. So, the influence functions and the command games are equivalent if the set of commandable players is equal to the set of followers. For some influence functions introduced in [10], we define the equivalent command games. In particular, we show that not for all influence functions compatible command games exist.

Moreover, we propose a more general definition of the influence index and, as already mentioned, we show that under some assumptions, some power indices which can be used in the command games, can be expressed through the weighted influence indices under the considered command influence functions. It is interesting to remark that both the Shapley-Shubik index and the Banzhaf index are equal to a difference between the weighted influence indices under some influence functions, and the only difference between these two power indices lies in the weights for the influence indices.

The structure of the paper is the following. Definitions of power indices we refer to in the paper, and the framework of the command games are briefly presented in Section 2. The latter model of the relationship we search for, that is, the influence model, is summarized in Section 3. The core of the paper is presented in Sections 4 and 5, where the relation between the influence model and the command games is investigated. In Section 4, we define several influence functions which are equivalent or compatible with the command games. We show relations between the weighted influence indices under the command influence functions and two well-known power indices, i.e., the Shapley-Shubik index and the (absolute) Banzhaf index. In Section 5, we define the equivalent command games for some influence functions introduced in [10]. In Section 6, the relations between the influence model and the command games are illustrated by an example mentioned in [14], i.e., by the Confucian model of society. In Section 7, we conclude. The paper contains also an appendix, in which we show relations between the weighted influence indices and some other power indices, i.e., the Coleman indices and the König-Bräuninger index. 


\section{Basic definitions and notations}

\subsection{Power indices}

First, we introduce several notations for convenience. Cardinality of sets $S, T, \ldots$ will be denoted by the corresponding lower case $s, t, \ldots$ We omit braces for sets, e.g., $\{k, m\}$, $N \backslash\{j\}, S \cup\{j\}$ will be written $k m, N \backslash j, S \cup j$, etc.

A $(0,1)$-game is a pair $(N, v)$, where $N=\{1, \ldots, n\}$ is the set of players, and a function $v: 2^{N} \rightarrow\{0,1\}$ satisfying $v(\emptyset)=0$ is the characteristic function. A nonempty subset of $N$ is called a coalition. A simple game is a $(0,1)$-game such that $v$ is not identically equal to 0 and is monotonic, i.e., $v(S) \leq v(T)$ whenever $S \subseteq T$. A coalition $S$ is winning if $v(S)=1$, and is loosing if $v(S)=0$. A game is superadditive if $v(S \cup T) \geq v(S)+v(T)$ whenever $S \cap T=\emptyset$. Let $S G_{n}$ denote the set of all simple superadditive $n$-person games. Player $k$ is a swing in a winning coalition $S$ if his removal from the coalition makes it loosing, i.e., if $v(S)=1$ and $v(S \backslash k)=0$. A minimal winning coalition is a winning coalition in which all players are swings.

A power index is a function $\phi: S G_{n} \rightarrow \mathbb{R}^{n}$ which assigns to each $(N, v) \in S G_{n}$ a vector $\phi(N, v)=\left(\phi_{1}(N, v), \ldots, \phi_{n}(N, v)\right)$.

The Shapley-Shubik index [23] of player $k \in N$ in a game $(N, v)$ is defined by

$$
S h_{k}(N, v)=\sum_{S \subseteq N: k \in S} \frac{(n-s) !(s-1) !}{n !}(v(S)-v(S \backslash k)) .
$$

The non-normalized Banzhaf index (the absolute Banzhaf index, [2]) of player $k \in N$ in a game $(N, v)$ is defined by

$$
B z_{k}(N, v)=\frac{1}{2^{n-1}} \sum_{S \subseteq N: k \in S}(v(S)-v(S \backslash k)),
$$

and the normalized Banzhaf index $\widetilde{B z}$ is given by

$$
\widetilde{B z}_{k}(N, v)=\frac{B z_{k}(N, v)}{\sum_{j \in N} B z_{j}(N, v)} .
$$

The Coleman 'power of a collectivity to act' $[3,4]$ in a game $(N, v)$ is defined by

$$
A(N, v)=\frac{\sum_{S \subseteq N} v(S)}{2^{n}} .
$$

The Coleman index 'to prevent action' [3, 4$]$ of player $k \in N$ in a game $(N, v)$ is defined by

$$
\operatorname{Col}_{k}^{P}(N, v)=\frac{\sum_{S \subseteq N: k \in S}(v(S)-v(S \backslash k))}{\sum_{S \subseteq N} v(S)} .
$$
by

The Coleman index 'to initiate action' [3,4] of player $k \in N$ in a game $(N, v)$ is given

$$
\operatorname{Col}_{k}^{I}(N, v)=\frac{\sum_{S \subseteq N: k \notin S}(v(S \cup k)-v(S))}{2^{n}-\sum_{S \subseteq N} v(S)} .
$$

The König-Bräuninger inclusiveness index [16] of player $k \in N$ in a game $(N, v)$ is given by

$$
K B_{k}(N, v)=\frac{\sum_{S \subseteq N: k \in S} v(S)}{\sum_{S \subseteq N} v(S)} .
$$




\subsection{Command games}

We recapitulate briefly the main concepts concerning the command games introduced by $\mathrm{Hu}$ and Shapley $[13,14]$. Let $N=\{1, \ldots, n\}$ be the set of players (voters). For $k \in N$ and $S \subseteq N \backslash k:$

- $S$ is a boss set for $k$ if $S$ determines the choice of $k$;

$-S$ is an approval set for $k$ if $k$ can act with an approval of $S$.

It is assumed that any superset (in $N \backslash k$ ) of a boss set is a boss set.

For each $k \in N$, a simple game $\left(N, \mathcal{W}_{k}\right)$ is built, called the command game for $k$, where the set of winning coalitions is

$\mathcal{W}_{k}:=\{S \mid S$ is a boss set for $k\} \cup\{S \cup k \mid S$ is a boss or approval set for $k\}$.

We can recover the boss sets for $k$

$$
\text { Boss }_{k}=\left\{S \subseteq N \backslash k \mid S \in \mathcal{W}_{k}\right\}=\mathcal{W}_{k} \cap 2^{N \backslash k}
$$

and the approval sets for $k$

$$
A p p_{k}=\left\{S \subseteq N \backslash k \mid S \cup k \in \mathcal{W}_{k} \text { but } S \notin \mathcal{W}_{k}\right\}
$$

We have Boss $_{k} \cap A p p_{k}=\emptyset$. In particular, if $A p p_{k}=2^{N \backslash k}$, then $k$ is called a free agent: he needs no approval (since $\emptyset \in A p p_{k}$ ), and nobody can boss him (since $\operatorname{Boss}_{k}=\emptyset$ ). If $A p p_{k}=\emptyset$, then $k$ is called a $\operatorname{cog}$.

Given the command games $\left\{\left(N, \mathcal{W}_{k}\right) \mid k \in N\right\}$, for any coalition $S \subseteq N$, the command function $\omega(S)$ is defined as the set of all members that are 'commandable' by $S$ :

$$
\omega(S):=\left\{k \in N \mid S \in \mathcal{W}_{k}\right\}
$$

In [13], it is shown that $\omega(\emptyset)=\emptyset, \omega(N)=N$, and $\omega(S) \subseteq \omega\left(S^{\prime}\right)$ whenever $S \subset S^{\prime}$.

In $[13,14]$, the authors define an authority distribution $\pi=\left(\pi_{1}, \ldots, \pi_{n}\right)$ over an organization $\left(N,\left\{\left(N, \mathcal{W}_{k}\right) \mid k \in N\right\}\right)$, and they create the power transition matrix of the organization, which is the stochastic matrix $P=[P(j, k)]_{j, k=1}^{n}$ such that

$$
P(j, k):=S h_{k}\left(N, \mathcal{W}_{j}\right)
$$

and $S h_{k}\left(N, \mathcal{W}_{j}\right)$ is the Shapley-Shubik index of player $k$ in the command game for player $j$. Hence, $P(j, k)$ represents the power of player $k$ to "influence" player $j$. They use a Markov chain to describe the organization's long-run authority $\pi$. The authority distribution $\pi$ is assumed to satisfy the authority equilibrium equation given by

$$
\pi=\pi P, \text { i.e., } \pi_{k}=\sum_{j \in N} \pi_{j} P(j, k), \forall k \in N
$$




\section{The influence model}

\subsection{Direct versus opposite influence}

In this section, the main concepts introduced in [10] are summarized. Let $N:=\{1, \ldots, n\}$ be the set of players (agents, actors, voters). The players are to make a yes-no decision. Each player has an inclination either to say 'yes' (denoted by +1 ) or 'no' (denoted by $-1)$. Let $i=\left(i_{1}, \ldots, i_{n}\right)$ denote an inclination vector and $I:=\{-1,+1\}^{n}$ be the set of all inclination vectors. In particular, $(1,1, \ldots, 1) \in I$ and $(-1,-1, \ldots,-1) \in I$ will be denoted by $1_{N}$ and $-1_{N}$, respectively. Players may influence each other, and as a consequence of the influence, the final decision of a player may be different from his original inclination. Each inclination vector $i \in I$ is therefore transformed into a decision vector $B i=\left((B i)_{1}, \ldots,(B i)_{n}\right)$, where $B: I \rightarrow I$ is the influence function. Let $\mathcal{B}$ denote the set of all influence functions.

We are interested in measuring the influence that a coalition of players with the same inclination has on an agent outside that coalition. We distinguish between a direct influence and an opposite influence. In the first case, the player's inclination is different from the inclination of that coalition, but the player's decision is the same as the inclination of the coalition. In the latter case, the player's inclination coincides with the inclination of the coalition, but by a kind of reactive behavior, his decision is different from the inclination of the coalition. To formalize, we introduce for any $S \subseteq N$ the set

$$
I_{S}:=\left\{i \in I \mid \forall k, j \in S\left[i_{k}=i_{j}\right]\right\}
$$

and $I_{k}:=I$, for any $k \in N$. We denote by $i_{S}$ the value $i_{k}$ for some $k \in S, i \in I_{S}$. For each $S \subseteq N$ and $j \in N$, we define the set $I_{S \rightarrow j}$ of all inclination vectors of potential direct influence of $S$ on $j$, and the set $I_{S \rightarrow j}^{*}(B)$ of all inclination vectors of observed direct influence of $S$ on $j$ under given $B \in \mathcal{B}$ :

$$
\begin{gathered}
I_{S \rightarrow j}:=\left\{i \in I_{S} \mid i_{j}=-i_{S}\right\} \\
I_{S \rightarrow j}^{*}(B):=\left\{i \in I_{S \rightarrow j} \mid(B i)_{j}=i_{S}\right\} .
\end{gathered}
$$

Similarly, for each $S \subseteq N$ and $j \in N$ we introduce the set $I_{S \rightarrow j}^{o p}$ of all inclination vectors of potential opposite influence, and the set $I_{S \rightarrow j}^{* o p}(B)$ of all inclination vectors of observed opposite influence of a coalition on a player:

$$
\begin{gathered}
I_{S \rightarrow j}^{o p}:=\left\{i \in I_{S} \mid i_{j}=i_{S}\right\} \\
I_{S \rightarrow j}^{* o p}(B):=\left\{i \in I_{S \rightarrow j}^{o p} \mid(B i)_{j}=-i_{S}\right\} .
\end{gathered}
$$

For each $S \subseteq N$ and $j \in N \backslash S$, we introduce a set of weights $\alpha_{i}^{S \rightarrow j} \in[0,1]$, for all inclination vectors $i \in I_{S \rightarrow j}$ (or $I_{S \rightarrow j}^{o p}$ ). The weight $\alpha_{i}^{S \rightarrow j}$ represents how much the inclination vector $i$ is important when computing the influence of $S$ over $j$ (other intepretations are possible, e.g., probability of occurrence, etc.). We assume that for each $S \subseteq N$ and $j \in N \backslash S$, there exists $i \in I_{S \rightarrow j}$ such that $\alpha_{i}^{S \rightarrow j}>0$, and there exists $i \in I_{S \rightarrow j}^{o p}$ such that $\alpha_{i}^{S \rightarrow j}>0$. Moreover, as stated in [10], we impose for simplicity the symmetry assumption that $\alpha_{i}^{S \rightarrow j}$ depends solely on the number of players with the same inclination as players of $S$ under $i \in I_{S}$ (including the players from $S$, but excluding player $j$ ). Apart from 
these assumptions, no other restrictions are needed. In particular, we do not impose any normalization condition, although such a condition could be added, if the weights convey some special meaning, e.g., probabilities, etc. Determining $\alpha_{i}^{S \rightarrow j}$ depends on which inclination vectors (viewed as particular 'situations') we want to take into account or to emphasize (or which are more likely to occur) for computing the influence index. If, for example, we consider a model which fully takes into account any possibility of influence, all weights are always equal to 1 . If, on the other hand, we take into account only those situations (i.e., inclination vectors) in which all agents outside $S \cup j$ have the inclination different from the inclination of $S$, we assign 1 to $\alpha_{i}^{S \rightarrow j}$ under such $i$ 's, and 0 to $\alpha_{i}^{S \rightarrow j}$ in all remaining cases. In [10] we consider also another way of sharing the ability to influence, in which, given $S$ and $j$, two inclination vectors with equal numbers of players having the same inclination as the inclination of $S$ are treated equally, but the maximal weight 1 is divided by the number of possible coalitions that could be 'responsible' for the influence.

Given $B \in \mathcal{B}$, for each $S \subseteq N, j \in N \backslash S$, the weighted direct influence index of coalition $S$ on player $j$ under $B$ is defined as

$$
d_{\alpha}(B, S \rightarrow j):=\frac{\sum_{i \in I_{S \rightarrow j}^{*}(B)} \alpha_{i}^{S \rightarrow j}}{\sum_{i \in I_{S \rightarrow j}} \alpha_{i}^{S \rightarrow j}} \in[0,1],
$$

and the weighted opposite influence index of coalition $S$ on player $j$ under $B$ is defined as

$$
d_{\alpha}^{o p}(B, S \rightarrow j):=\frac{\sum_{i \in I_{S \rightarrow j}^{* o p}(B)} \alpha_{i}^{S \rightarrow j}}{\sum_{i \in I_{S \rightarrow j}^{o p}} \alpha_{i}^{S \rightarrow j}} \in[0,1] .
$$

When measuring direct influence of coalition $S$ on player $j$, the point of departure is the set of all inclination vectors of potential direct influence of $S$ on $j$, and consequently, in the denominator of the weighted direct influence index of $S$ on $j$, we have the weighted sum over such inclination vectors. Furthermore, we consider only those inclination vectors of potential direct influence that are the vectors of observed direct influence, and in the numerator of the index we have the weighted sum over such inclination vectors of observed direct influence. The definition of the weighted opposite influence index is analogous, except that instead of inclination vectors of (potential or observed) direct influence, we consider inclination vectors of (potential or observed) opposite influence.

One of the key concepts in the influence model is the concept of a follower of a given coalition, that is, a voter who always follows the inclination of the coalition in question. Let $\emptyset \neq S \subseteq N$ and $B \in \mathcal{B}$. The set $F_{B}(S)$ of followers of $S$ under $B$ is therefore defined as

$$
F_{B}(S):=\left\{j \in N \mid \forall i \in I_{S}\left[(B i)_{j}=i_{S}\right]\right\} .
$$

We put $F_{B}(\emptyset):=\emptyset$. We have, in particular,

$$
d_{\alpha}(B, S \rightarrow j)=1, \forall j \in F_{B}(S) \backslash S .
$$

Assume $F_{B}$ is not identically the empty set. We define the kernel of $B$, that is, the set of 'true' influential coalitions. A set $S$ belongs to the kernel of $B$ if $S$ has a non-empty set of followers under $B$, but any of its proper subsets has no follower.

$$
\mathcal{K}(B):=\left\{S \in 2^{N} \mid F_{B}(S) \neq \emptyset \text {, and } S^{\prime} \subset S \Rightarrow F_{B}\left(S^{\prime}\right)=\emptyset\right\} .
$$




\section{$3.2 \quad$ A general case of influence}

Next, we generalize the concept of influence to be able to comprise all imaginable cases of influence, in particular, the direct influence, the opposite influence, an influence of a coalition on its member, etc. The point of departure will be the set $I_{S}$ defined in (10). In other words, we assume that a coalition may influence a player only in situations when all members of the coalition have the same inclination. Furthermore, an observed influence of a coalition $S$ on a player $j$, which is now characterized by $\lambda \in\left\{-i_{j}, i_{S},-i_{S},+1,-1\right\}$, takes place if $(B i)_{j}=\lambda$. Hence, the set $I_{S \rightarrow j, \lambda}(B)$ of all inclination vectors of influence of $S$ on $j$ under $B$ is defined as

$$
I_{S \rightarrow j, \lambda}(B):=\left\{i \in I_{S} \mid(B i)_{j}=\lambda\right\} .
$$

Definition 1 Given $B \in \mathcal{B}$, for each $S \subseteq N, j \in N$, the weighted influence index of coalition $S$ on player $j$ under $B$ is defined as

$$
\psi_{\alpha, \lambda}(B, S \rightarrow j):=\frac{\sum_{i \in I_{S \rightarrow j, \lambda}(B)} \alpha_{i}^{S \rightarrow j}}{\sum_{i \in I_{S}} \alpha_{i}^{S \rightarrow j}}
$$

The set $I_{S \rightarrow j, \lambda}(B)$ captures several kinds of decisions made by the influenced player $j$, depending on $\lambda$. In other words, we can consider different definitions of the influence. If, for instance, $\lambda=i_{S}$, then by the influence of $S$ we mean following the inclination of that coalition $S$, and consequently, when measuring the influence we record all the situations in which player $j$ decides according to the inclination of coalition $S$. In such situations, for example, $I_{S \rightarrow j, \lambda=i_{S}}(B)=I_{S}$ means that player $j$ is a follower of coalition $S$ under $B$, and the weighted influence index $\psi_{\alpha, \lambda=i_{S}}(B, S \rightarrow j)=1$. While under $\lambda=-i_{S}$ we are interested in all the situations in which player $j$ decides differently from the inclination of coalition $S$, setting $\lambda=-i_{j}$ means that an influenced player is defined as a player whose decision is different from his inclination. In particular, the weighted direct influence index is recovered as follows:

$$
\psi_{\alpha, \lambda}(B, S \rightarrow j)=d_{\alpha}(B, S \rightarrow j) \quad \text { if } \quad \lambda=-i_{j} \quad \text { and } \quad \alpha_{i}^{S \rightarrow j}=0 \quad \text { for } \quad i_{j}=i_{S},
$$

and the weighted opposite influence index is recovered as:

$$
\psi_{\alpha, \lambda}(B, S \rightarrow j)=d_{\alpha}^{o p}(B, S \rightarrow j) \quad \text { if } \quad \lambda=-i_{j} \quad \text { and } \quad \alpha_{i}^{S \rightarrow j}=0 \quad \text { for } \quad i_{j}=-i_{S} .
$$

The essence of both the direct influence and the opposite influence is that the decision of an influenced player is different from his original inclination $\left(\lambda=-i_{j}\right)$. The only difference between these two types of influence lies in the inclination vectors we consider: while in the direct influence we observe only situations in which the inclinations of a player and an influencing coalition are different from each other $\left(\alpha_{i}^{S \rightarrow j}=0\right.$ for $\left.i_{j}=i_{S}\right)$, in case of the opposite influence we take into account only situations in which the inclinations of a player and an influencing coalition are the same $\left(\alpha_{i}^{S \rightarrow j}=0\right.$ for $\left.i_{j}=-i_{S}\right)$.

The set of followers and the kernel are defined like in (11) and (12), respectively. 


\subsection{Influence functions}

We recapitulate some influence functions defined in [10] that we refer to in the present paper.

(i) The Majority function - Let $n \geq t>\left\lfloor\frac{n}{2}\right\rfloor$, and introduce for any $i \in I$ the set $i^{+}:=\left\{k \in N \mid i_{k}=+1\right\}$. The majority influence function $\mathrm{Maj}^{[t]} \in \mathcal{B}$ is defined by

$$
\operatorname{Maj}^{[t]} i:=\left\{\begin{array}{ll}
1_{N}, & \text { if }\left|i^{+}\right| \geq t \\
-1_{N}, & \text { if }\left|i^{+}\right|<t
\end{array}, \quad \forall i \in I\right.
$$

which simply means that if a majority of players has an inclination +1 , then all players decide +1 , and if not, all players decide -1 . We have for each $S \subseteq N$ :

$$
\begin{gathered}
F_{\mathrm{Maj}^{[t]}}(S)= \begin{cases}N, & \text { if } s \geq t \\
\emptyset, & \text { if } s<t\end{cases} \\
\mathcal{K}\left(\mathrm{Maj}^{[t]}\right)=\{S \subseteq N|| S \mid=t\} .
\end{gathered}
$$

(ii) The Guru function - Let $\widetilde{k} \in N$ be a particular player called the guru. The guru influence function Gur $^{[\widetilde{k}]} \in \mathcal{B}$ is defined by

$$
\left(\operatorname{Gur}^{[\widetilde{k}]} i\right)_{j}=i_{\widetilde{k}}, \quad \forall i \in I, \quad \forall j \in N
$$

that is, when a guru exists, every player always follows the guru. We have for each $S \subseteq N$ :

$$
\begin{gathered}
F_{\text {Gur }^{[\tilde{k}]}}(S)= \begin{cases}N, & \text { if } \widetilde{k} \in S \\
\emptyset, & \text { if } \widetilde{k} \notin S\end{cases} \\
\mathcal{K}\left(\text { Gur }^{[\widetilde{k}]}\right)=\{\widetilde{k}\} .
\end{gathered}
$$

(iii) The identity function $\mathrm{Id} \in \mathcal{B}$ is defined by

$$
\operatorname{ld} i=i, \quad \forall i \in I,
$$

and simply depicts the absence of any influence. We have for each $S \subseteq N, F_{\text {Id }}(S)=S$. The kernel is $\mathcal{K}(\mathrm{Id})=\{\{k\}, k \in N\}$.

(iv) The reversal function $-\mathrm{Id} \in \mathcal{B}$ is simply the opposite of the identity function:

$$
-\mathrm{Id} i=-i, \quad \forall i \in I
$$

We have for each $S \subseteq N, F_{- \text {Id }}(S)=\emptyset$. The kernel is $\mathcal{K}(-$ ld $)=\emptyset$. 


\section{Command influence functions}

We can apply our model of influence to the framework of command games. First, we propose several influence functions, called here the command influence functions that are compatible with the command games, in the sense that each commandable player for a coalition in the command game is a follower of that coalition under the command influence function. In a sense, the command influence functions explain the command games, specifying how exactly players decide according to their boss and approval sets. A particular case of the influence functions which are compatible with the command games are the functions that are equivalent to the command games. A command influence function is equivalent to the set of command games if it is compatible with this set of command games, and additionally each follower of a coalition under the command influence function is also a commandable player for that coalition in the command games.

Definition 2 Let $\left\{\left(N, \mathcal{W}_{k}\right) \mid k \in N\right\}$ be a set of command games, $\omega(S)$ be a command function as defined in (7), and $F_{B}(S)$ denote the set of followers of $S$ under the influence function $B$. The influence function $B$ and the set of command games $\left\{\left(N, \mathcal{W}_{k}\right) \mid k \in N\right\}$ are compatible if

$$
\omega(S) \subseteq F_{B}(S), \forall S \subseteq N
$$

The influence function $B$ and the set of command games $\left\{\left(N, \mathcal{W}_{k}\right) \mid k \in N\right\}$ are equivalent if $\omega \equiv F_{B}$.

For each set of command games, a necessary condition for the existence of an influence function which is compatible with the command games is that for each player $k$, any two winning coalitions in the command game for $k$ have at least one common member.

Proposition 1 Let $\left\{\left(N, \mathcal{W}_{k}\right) \mid k \in N\right\}$ be a set of command games. If there exists an influence function which is compatible with this set of command games, then the following holds:

$$
\forall k \in N \forall S, S^{\prime} \in \mathcal{W}_{k}\left[S \cap S^{\prime} \neq \emptyset\right]
$$

Proof: Suppose that there exists an influence function $B$ which is compatible with the set of command games $\left\{\left(N, \mathcal{W}_{k}\right) \mid k \in N\right\}$, and the condition (21) does not hold. Hence, from (20), $\omega(S) \subseteq F_{B}(S)$, for each $S \subseteq N$. Moreover, since (21) does not hold, there exist $k \in N$, and $S, S^{\prime} \in \mathcal{W}_{k}$ such that $S \cap S^{\prime}=\emptyset$. Since $S, S^{\prime} \in \mathcal{W}_{k}$, we have $k \in \omega(S)$ and $k \in \omega\left(S^{\prime}\right)$, which by virtue of (20) implies $k \in F_{B}(S)$ and $k \in F_{B}\left(S^{\prime}\right)$. ¿From (11), $(B i)_{k}=i_{S}$ for each $i \in I_{S}$, and $(B i)_{k}=i_{S^{\prime}}$ for each $i \in I_{S^{\prime}}$. Take $i \in I_{S} \cap I_{S^{\prime}}$ such that $i_{S} \neq i_{S^{\prime}}$. Hence, we have $(B i)_{k}=i_{S} \neq i_{S^{\prime}}=(B i)_{k}$, a contradiction.

The context of the rest of this section is the following. First, for an arbitrary set of command games, we construct one compatible command influence function (Section 4.2), and two equivalent command influence functions (Sections 4.1 and 4.3). This means that our model of influence is broader than the framework of the command games. Moreover, while in $[13,14]$ the authors apply the Shapley-Subik index to the command games, in the present paper we apply several power indices (i.e., the ones recapitulated in Section 2.1) to the command games. We show that these power indices coincide with some expressions 
of the weighted influence indices under the command influence functions. In this section, we present the relations concerning the Shapley-Shubik and the Banzhaf index, and in the Appendix we show the relations concerning the Coleman indices and the KönigBräuninger index.

\subsection{An influence function with abstention}

The first command influence function we propose is related to an extended model of influence (see [9]), in which players have an ordered set of possible actions. In this model, each player has an inclination either to say 'yes' (denoted by +1 ) or 'no' (denoted by -1 ), that is, $I=\{-1,+1\}^{n}$, but he has three options for making his decision: 'yes', 'no', or 'to abstain' (denoted by 0 ), that is, $B(I) \subseteq\{-1,0,+1\}^{n}$. The command influence function is defined as follows.

Definition 3 Given a set of command games $\left\{\left(N, \mathcal{W}_{k}\right) \mid k \in N\right\}$, the command influence function Com $\in \mathcal{B}$ is defined for each $k \in N$ and $i \in I$ by

$$
(\operatorname{Com} i)_{k}:= \begin{cases}+1, & \text { if }\left\{j \in N \mid i_{j}=+1\right\} \in \mathcal{W}_{k} \\ -1, & \text { if }\left\{j \in N \mid i_{j}=-1\right\} \in \mathcal{W}_{k} \\ 0, & \text { otherwise }\end{cases}
$$

By Proposition 1, Com is well defined. According to the command influence function Com, for each voter $k$ and each inclination vector, if all players with the same inclination forms a winning coalition in his command game, the voter $k$ follows the inclination of this winning coalition. Otherwise, that is, if none of the two coalitions with all members having the same inclination is winning, the voter $k$ simply abstains.

Proposition 2 Let $\left\{\left(N, \mathcal{W}_{k}\right) \mid k \in N\right\}$ be a set of command games, and $\omega(S)$ be a command function as defined in (7). We have

$$
F_{\text {Com }}(S)=\omega(S), \forall S \subseteq N
$$

where $F_{\mathrm{Com}}(S)$ is the set of followers of $S$ under the command influence function Com.

Proof: $\quad F_{\text {Com }}(\emptyset)=\emptyset=\omega(\emptyset)$. Take an arbitrary nonempty $S \subseteq N$. Suppose that $F_{\text {Com }}(S) \nsubseteq \omega(S)$. Hence, there exists $k \in F_{\text {Com }}(S)$ such that $k \notin \omega(S)$, and therefore $(\operatorname{Com} i)_{k}=i_{S}$ for all $i \in I_{S}$, and $S \notin \mathcal{W}_{k}$. Take $i \in I_{S}$ such that $i_{S}=+1$, and $i_{j}=-1$ for each $j \notin S$. Hence, $(\operatorname{Com} i)_{k}=i_{S}=+1$, but since $S \notin \mathcal{W}_{k}$ we have also either $(\operatorname{Com} i)_{k}=-1$ or $(\operatorname{Com} i)_{k}=0$, a contradiction.

Suppose now that $\omega(S) \nsubseteq F_{\text {Com }}(S)$. Hence, there exists $k \in \omega(S)$ such that $k \notin F_{\text {Com }}(S)$. This means that $S \in \mathcal{W}_{k}$, and there exists $i \in I_{S}$ such that $(\operatorname{Com} i)_{k} \neq i_{S}$. However, by $(22),(\operatorname{Com} i)_{k}=i_{S}$ which contradicts $(\operatorname{Com} i)_{k} \neq i_{S}$.

Consequently, by virtue of (12) and (23), the kernel of Com is given by:

$$
\mathcal{K}(\mathrm{Com})=\left\{S \in 2^{N} \mid \omega(S) \neq \emptyset \text {, and } S^{\prime} \subset S \Rightarrow \omega\left(S^{\prime}\right)=\emptyset\right\} .
$$


Remark 1 By virtue of (23), for each set of command games $\left\{\left(N, \mathcal{W}_{k}\right) \mid k \in N\right\}$, the command influence function $\mathrm{Com} \in \mathcal{B}$ defined in (22) is equivalent to this set of command games.

Proposition 3 Let $\left\{\left(N, \mathcal{W}_{j}\right) \mid j \in N\right\}$ be a set of command games, and Com be the command influence function defined in (22). Then for each $j, k \in N$

$$
S h_{k}\left(N, \mathcal{W}_{j}\right)=\psi_{\alpha^{(S h)}, \lambda=i_{k}}(\operatorname{Com}, k \rightarrow j)-\psi_{\alpha^{(S h)}, \lambda=-i_{k}}(\operatorname{Com}, k \rightarrow j)
$$

where $S h_{k}\left(N, \mathcal{W}_{j}\right)$ is the Shapley-Shubik index of player $k$ in the command game for $j$, $\psi_{\alpha, \lambda}$ is the weighted influence index defined in (13), and for each $i \in I$

$$
\alpha_{i}^{(S h) k \rightarrow j}= \begin{cases}\frac{1}{n\left(\begin{array}{c}
n-1 \\
|i|-1
\end{array}\right)}, & \text { if } i_{k}=+1 \\
\frac{1}{n\left(\begin{array}{c}
n-1 \\
n-|i|-1
\end{array}\right)}, & \text { if } i_{k}=-1\end{cases}
$$

and $|i|:=\left|\left\{m \in N \mid i_{m}=+1\right\}\right|$.

Proof: By a coalition $S$ we mean the set of players with the same inclination $i_{S}$, while all players outside $S$ have the inclination $-i_{S}$. Consequently, two coalitions are formed under each inclination vector: a coalition of players with positive inclination, and a coalition of players with negative inclination. We have: $k \in S$ iff $i_{k}=i_{S}$. Moreover, by (22), given $\left(N, \mathcal{W}_{j}\right): v(S)=1$ iff $S \in \mathcal{W}_{j}$ iff $(\operatorname{Com} i)_{j}=i_{S}$ for each $i \in I_{S}$ such that $i_{m}=-i_{S}$ for each $m \notin S$. From (1), we have

$$
\begin{aligned}
S h_{k}\left(N, \mathcal{W}_{j}\right) & =\sum_{S \subseteq N: k \in S} \frac{(n-s) !(s-1) !}{n !}(v(S)-v(S \backslash k)) \\
& =\sum_{S \subseteq N: k \in S} \frac{(n-s) !(s-1) !}{n !} v(S)-\sum_{S \subseteq N: k \notin S} \frac{(n-s-1) ! s !}{n !} v(S) \\
& =\sum_{S \subseteq N: k \in S} \frac{v(S)}{n\left(\begin{array}{c}
n-1 \\
s-1
\end{array}\right)}-\sum_{S \subseteq N: k \notin S} \frac{v(S)}{n\left(\begin{array}{c}
n-1 \\
n-s-1
\end{array}\right)} \\
& =\sum_{S \in \mathcal{W}_{j}: k \in S} \frac{1}{n\left(\begin{array}{l}
n-1 \\
s-1
\end{array}\right)}-\sum_{S \in \mathcal{W}_{j}: k \notin S} \frac{1}{n\left(\begin{array}{c}
n-1 \\
n-s-1
\end{array}\right)}=\frac{1}{2}\left[\sum_{i \in I_{k}^{+}} \alpha_{i}^{(S h) k \rightarrow j}-\sum_{i \in I_{k}^{-}} \alpha_{i}^{(S h) k \rightarrow j}\right]
\end{aligned}
$$

with

$$
I_{k}^{+}:=\left\{i \in I \mid(\operatorname{Com} i)_{j}=i_{k}\right\}, \quad I_{k}^{-}:=\left\{i \in I \mid(\operatorname{Com} i)_{j}=-i_{k}\right\} .
$$

Indeed,

$$
\sum_{i \in I_{k}^{+}} \alpha_{i}^{(S h) k \rightarrow j}=\sum_{i \in I_{k}^{+}: i_{k}=+1} \frac{1}{n\left(\begin{array}{c}
n-1 \\
|i|-1
\end{array}\right)}+\sum_{i \in I_{k}^{+}: i_{k}=-1} \frac{1}{n\left(\begin{array}{c}
n-1 \\
n-|i|-1
\end{array}\right)} .
$$

By definition of Com $i$, we have $\left[i \in I_{k}^{+} \wedge i_{k}=+1\right]$ iff $\left[i^{+}:=\left\{m \in N \mid i_{m}=+1\right\} \in\right.$ $\left.\mathcal{W}_{j} \wedge k \in i^{+}\right]$. Similarly, $\left[i \in I_{k}^{+} \wedge i_{k}=-1\right]$ iff $\left[i^{-}:=\left\{m \in N \mid i_{m}=-1\right\} \in \mathcal{W}_{j} \wedge k \in i^{-}\right]$. Denoting winning coalitions by $S$, we obtain

$$
\sum_{i \in I_{k}^{+}} \alpha_{i}^{(S h) k \rightarrow j}=\sum_{S \in \mathcal{W}_{j}: k \in S} \frac{1}{n\left(\begin{array}{c}
n-1 \\
s-1
\end{array}\right)}+\sum_{S \in \mathcal{W}_{j}: k \in S} \frac{1}{n\left(\begin{array}{c}
n-1 \\
s-1
\end{array}\right)}=2 \sum_{S \in \mathcal{W}_{j}: k \in S} \frac{1}{n\left(\begin{array}{c}
n-1 \\
s-1
\end{array}\right)}
$$


Similarly, we obtain

$$
\begin{gathered}
\sum_{i \in I_{k}^{-}} \alpha_{i}^{(S h) k \rightarrow j}=2 \sum_{S \in \mathcal{W}_{j}: k \notin S} \frac{1}{n\left(\begin{array}{c}
n-1 \\
n-s-1
\end{array}\right)} . \\
\sum_{i: i_{k}=+1} \alpha_{i}^{(S h) k \rightarrow j}=\sum_{i: i_{k}=+1} \frac{1}{n\left(\begin{array}{c}
n-1 \\
|i|-1
\end{array}\right)}=\sum_{|i|=1}^{n} \frac{1}{n\left(\begin{array}{c}
n-1 \\
|i|-1
\end{array}\right)}\left(\begin{array}{c}
n-1 \\
|i|-1
\end{array}\right)=1 \\
\sum_{i: i_{k}=-1} \alpha_{i}^{(S h) k \rightarrow j}=\sum_{i: i_{k}=-1} \frac{1}{n\left(\begin{array}{c}
n-1 \\
n-|i|-1
\end{array}\right)}=\sum_{|i|=0}^{n-1} \frac{1}{n\left(\begin{array}{c}
n-1 \\
n-|i|-1
\end{array}\right)}\left(\begin{array}{c}
n-1 \\
|i|
\end{array}\right)=1,
\end{gathered}
$$

and therefore

$$
\begin{gathered}
\sum_{i \in I} \alpha_{i}^{(S h) k \rightarrow j}=2 \\
S h_{k}\left(N, \mathcal{W}_{j}\right)=\frac{1}{2}\left[\sum_{i \in I_{k}^{+}} \alpha_{i}^{(S h) k \rightarrow j}-\sum_{i \in I_{k}^{-}} \alpha_{i}^{(S h) k \rightarrow j}\right]=\frac{\sum_{i \in I_{k}^{+}} \alpha_{i}^{(S h) k \rightarrow j}}{\sum_{i \in I} \alpha_{i}^{(S h) k \rightarrow j}}-\frac{\sum_{i \in I_{k}^{-}} \alpha_{i}^{(S h) k \rightarrow j}}{\sum_{i \in I} \alpha_{i}^{(S h) k \rightarrow j}} \\
=\psi_{\alpha^{(S h)}, \lambda=i_{k}}(\operatorname{Com}, k \rightarrow j)-\psi_{\alpha^{(S h)}, \lambda=-i_{k}}(\operatorname{Com}, k \rightarrow j),
\end{gathered}
$$

where $\psi_{\alpha, \lambda}$ is the weighted influence index defined in (13), and $\alpha^{(S h)}$ is given in (25).

Proposition 4 Let $\left\{\left(N, \mathcal{W}_{j}\right) \mid j \in N\right\}$ be a set of command games, and Com be the command influence function defined in (22). Then for each $j, k \in N$

$$
B z_{k}\left(N, \mathcal{W}_{j}\right)=\psi_{\alpha^{(B z)}, \lambda=i_{k}}(\operatorname{Com}, k \rightarrow j)-\psi_{\alpha^{(B z)}, \lambda=-i_{k}}(\operatorname{Com}, k \rightarrow j)
$$

where $B z_{k}\left(N, \mathcal{W}_{j}\right)$ is the Banzhaf index of player $k$ in the command game for $j, \psi_{\alpha, \lambda}$ is the weighted influence index defined in (13) with

$$
\alpha_{i}^{(B z) k \rightarrow j}=1, \forall i \in I
$$

Proof: The proof is similar to the one of Proposition 3. From (2), we have

$$
\begin{aligned}
B z_{k}\left(N, \mathcal{W}_{j}\right) & =\frac{1}{2^{n-1}} \sum_{S \subseteq N: k \in S}(v(S)-v(S \backslash k))=\frac{1}{2^{n-1}} \sum_{S \subseteq N: k \in S} v(S)-\frac{1}{2^{n-1}} \sum_{S \subseteq N: k \notin S} v(S) \\
& =\frac{1}{2^{n-1}}\left[\frac{\left|\left\{i \in I \mid(\operatorname{Com} i)_{j}=i_{k}\right\}\right|}{2}-\frac{\left|\left\{i \in I \mid(\operatorname{Com} i)_{j}=-i_{k}\right\}\right|}{2}\right] \\
& =\frac{\left|\left\{i \in I \mid(\operatorname{Com} i)_{j}=i_{k}\right\}\right|}{|I|}-\frac{\left|\left\{i \in I \mid(\operatorname{Com} i)_{j}=-i_{k}\right\}\right|}{|I|} \\
& =\psi_{\alpha^{(B z)}, \lambda=i_{k}}(\operatorname{Com}, k \rightarrow j)-\psi_{\alpha^{(B z)}, \lambda=-i_{k}}(\operatorname{Com}, k \rightarrow j)
\end{aligned}
$$

where $\psi_{\alpha, \lambda}$ is the weighted influence index defined in (13), and $\alpha^{(B z)}$ is given in (27).

What we show in Propositions 3 and 4 is that under a certain assumption, both the Shapley-Shubik index and the Banzhaf index of player $k$ in the command game for $j$ are 
equal to the difference between the weighted influence index in which player $j$ is said to be influenced by $k$ if he follows $k$, and the weighted influence index in which the influence of $k$ on $j$ means that player $j$ 's decision is opposite to the inclination of $k$. Both weighted influence indices are measured under the command influence function defined in (22). The difference between the results on these two power indices lies only in the weights for the influence indices: while for the Shapley-Shubik index the weights are defined in (25), the weights for the Banzhaf index are always equal to 1, as given in (27).

The results shown in Propositions 3 and 4 are not very surprising. The essential part of both the Shapley-Shubik and Banzhaf indices of player $k$ is a marginal contribution $(v(S)-v(S \backslash k))$ of $k$ in any coalition $S$ containing $k$. We assume that a player is a member of a coalition if his inclination coincides with the inclination of that coalition. Since a coalition $S$ is winning (that is, $v(S)=1$ ) in the command game for $j$ if the player $j$ decides according to the inclination of $S$, considering the marginal contributions of $k$ means considering a difference between the inclination vectors under which the player $j$ decides according to the inclination of $k$, and the inclination vectors under which $j$ 's decision is different from the inclination of $k$. This difference between the specific inclination vectors is captured by our weighted influence indices. In order to get the equity between the Shapley-Shubik index and the difference between the weighted influence indices, the weights need to be properly defined, and they are equal to the coefficients of the Shapley-Shubik index. Similar remark holds for the Banzhaf index.

\subsection{An influence function in which a player without a boss follows himself}

Next, we mention another command influence function which is compatible with the command games.

Definition 4 Given a set of command games $\left\{\left(N, \mathcal{W}_{k}\right) \mid k \in N\right\}$, the command influence function $\widetilde{\mathrm{Com}} \in \mathcal{B}$ is defined for each $k \in N$ and $i \in I$ by

$$
\widetilde{(\operatorname{Com} i)_{k}}:= \begin{cases}+1, & \text { if }\left\{j \in N \mid i_{j}=+1\right\} \in \mathcal{W}_{k} \\ -1, & \text { if }\left\{j \in N \mid i_{j}=-1\right\} \in \mathcal{W}_{k} . \\ i_{k}, & \text { otherwise }\end{cases}
$$

The command influence function $\widetilde{\text { Com }}$ is similar to the function Com defined in (22) with a difference that for each inclination vector, if none of the two coalitions with all members having the same inclination is winning, now instead of abstaining, the voter in question simply follows his own inclination. As a consequence, we do not have the equality between the sets $\omega(S)$ and $F_{\widetilde{\text { Com }}}(S)$ (as we had before between $\omega(S)$ and $F_{\text {Com }}(S)$ ), but the inclusion. For each coalition $S$, the set of followers of $S$ under $\widetilde{C o m}$ consists of all players that are commandable by $S$, and of those members $k$ of $S$ for which any winning coalition in the command game for $k$ contains at least one player from $S$.

Proposition 5 Let $\left\{\left(N, \mathcal{W}_{k}\right) \mid k \in N\right\}$ be a set of command games, and $\omega(S)$ be a command function as defined in (7). We have

$$
F_{\widetilde{\mathrm{Com}}}(S)=\omega(S) \cup\left\{k \in S \mid \forall S^{\prime} \in \mathcal{W}_{k}\left[S \cap S^{\prime} \neq \emptyset\right]\right\}, \forall S \subseteq N,
$$

where $F_{\widetilde{\mathrm{Com}}}(S)$ is the set of followers of $S$ under the command influence function $\widetilde{\mathrm{Com}}$. 
Proof: Suppose that $F_{\widetilde{\mathrm{Com}}}(S) \nsubseteq \Phi(S) \cup\left\{k \in S \mid \forall S^{\prime} \in \mathcal{W}_{k}\left[S \cap S^{\prime} \neq \emptyset\right]\right\}$ for a certain $S \subseteq N$. Hence, there exists $k \in F_{\widetilde{\text { Com }}}(S)$ such that $k \notin \omega(S)$, and either $k \notin S$ or $\left(k \in S\right.$, but there exists $S^{\prime} \in \mathcal{W}_{k}$ such that $\left.S \cap S^{\prime}=\emptyset\right)$. Consequently, since $k \in F_{\widetilde{\mathrm{Com}}}(S)$ and $k \notin \omega(S)$, we have $(\widetilde{\operatorname{Com}} i)_{k}=i_{S}$ for all $i \in I_{S}$, and $S \notin \mathcal{W}_{k}$. Take $i \in I_{S}$ such that $i_{S}=+1$, and $i_{j}=-1$ for all $j \notin S$. Hence, $(\widetilde{\operatorname{Com} i})_{k}=i_{S}=+1$. If $k \notin S$, then $(\widetilde{\operatorname{Com} i})_{k}=i_{S}=+1$ and $\left.\widetilde{(\operatorname{Com} i}\right)_{k}=-1$, contradiction. Suppose that $k \in S$ and there is $S^{\prime} \in \mathcal{W}_{k}$ such that $S \cap S^{\prime}=\emptyset$. Hence, $i_{S^{\prime}}=-1$, and therefore $(\widetilde{\operatorname{Com} i})_{k}=+1$ and $(\widetilde{\mathrm{Com} i})_{k}=-1$, a contradiction.

Suppose now that $\omega(S) \cup\left\{k \in S \mid \forall S^{\prime} \in \mathcal{W}_{k}\left[S \cap S^{\prime} \neq \emptyset\right]\right\} \nsubseteq \subseteq F_{\widetilde{\text { Com }}}(S)$ for a certain $S \subseteq N$. Hence, there exists $k$ such that $k \notin F_{\widetilde{\mathrm{Com}}}(S)$, and either $k \in \omega(S)$ or $(k \in S$ and for all $\left.S^{\prime} \in \mathcal{W}_{k}, S \cap S^{\prime} \neq \emptyset\right)$. Since $k \notin F_{\widetilde{\operatorname{Com}}}(S)$, there exists $i \in I_{S}$ such that $(\widetilde{\operatorname{Com} i})_{k}=-i_{S}$. If $k \in \omega(S)$, then $S \in \mathcal{W}_{k}$, and therefore $(\widetilde{\operatorname{Com}} i)_{k}=i_{S}$, a contradiction. Suppose then $k \in S$ and for all $S^{\prime} \in \mathcal{W}_{k}, S \cap S^{\prime} \neq \emptyset$. Hence, in particular, $i_{k}=i_{S}$. If there is $S^{\prime} \in \mathcal{W}_{k}$ such that $i \in I_{S^{\prime}}$, then $(\widetilde{\operatorname{Com} i})_{k}=i_{S^{\prime}}=i_{S}$, a contradiction. If $i \notin I_{S^{\prime}}$ for all $S^{\prime} \in \mathcal{W}_{k}$, then $\widetilde{(\operatorname{Com} i})_{k}=i_{k}=i_{S}$, a contradiction.

Remark 2 By virtue of (29), for each set of command games $\left\{\left(N, \mathcal{W}_{k}\right) \mid k \in N\right\}$, the command influence function $\widetilde{\mathrm{Com}} \in \mathcal{B}$ defined in (28) is compatible with this set of command games.

\subsection{Non-symmetric influence functions}

Next, we propose a command influence function under which we treat a winning coalition as the set of potential yes-voters only.

Definition 5 Given a set of command games $\left\{\left(N, \mathcal{W}_{k}\right) \mid k \in N\right\}$, the command influence function $\overline{\mathrm{Com}} \in \mathcal{B}$ is defined by

$$
(\overline{\operatorname{Com}} i)_{k}:=\left\{\begin{array}{ll}
+1, & \text { if }\left\{j \in N \mid i_{j}=+1\right\} \in \mathcal{W}_{k} \\
-1, & \text { if }\left\{j \in N \mid i_{j}=+1\right\} \notin \mathcal{W}_{k}
\end{array}, \quad \forall i \in I, \quad \forall k \in N .\right.
$$

According to definition (30), for each player $k$, if the set of players with positive inclination creates a winning coalition in the command game for $k$, then $k$ decides 'yes', and if the set of players with positive inclination is not a winning coalition in the command game for $k$, then player $k$ 's decision is 'no'.

Note that we could define another command influence function in which instead of looking at the positive inclination of the players, we would consider only the negative inclination of the agents, and a winning coalition would be the set of potential no-voters only. Since the results of these two cases are similar, we study only the command influence function defined by (30).

Proposition 6 Let $\left\{\left(N, \mathcal{W}_{k}\right) \mid k \in N\right\}$ be a set of command games satisfying (21), and $\omega(S)$ be a command function as defined in (7). We have

$$
F_{\overline{\mathrm{Com}}}(S)=\omega(S), \forall S \subseteq N
$$

where $F_{\overline{\mathrm{Com}}}(S)$ is the set of followers of $S$ under the command influence function $\overline{\mathrm{Com}}$. 
Proof: $F_{\overline{\mathrm{Com}}}(\emptyset)=\emptyset=\omega(\emptyset)$. Take an arbitrary nonempty $S \subseteq N$. Suppose that $F_{\overline{\mathrm{Com}}}(S) \nsubseteq \omega(S)$. Hence, there exists $k \in F_{\overline{\mathrm{Com}}}(S)$ such that $k \notin \omega(S)$, and therefore $(\overline{\operatorname{Com}} i)_{k}=i_{S}$ for all $i \in I_{S}$, and $S \notin \mathcal{W}_{k}$. Take $i \in I_{S}$ such that $i_{S}=+1$ and $i_{m}=-1$ if $m \notin S$. Hence, $(\overline{\operatorname{Com}} i)_{k}=+1$ and $(\overline{\operatorname{Com}} i)_{k}=-1$, a contradiction.

Suppose now that $\omega(S) \nsubseteq F_{\overline{\mathrm{Com}}}(S)$. Hence, there exists $k \in \omega(S)$ such that $k \notin F_{\overline{\mathrm{Com}}}(S)$. This means that $S \in \mathcal{W}_{k}$ and there is $i \in I_{S}$ such that $(\overline{\operatorname{Com}} i)_{k}=-i_{S}$. Hence, if $i_{S}=+1$, then $(\overline{\operatorname{Com}} i)_{k}=-1$ and $(\overline{\operatorname{Com}} i)_{k}=+1$, because $S \in W_{k}$ implies $S^{\prime} \in W_{k}$ for any $S^{\prime} \supseteq S$, a contradiction. If $i_{S}=-1$, then $(\overline{\operatorname{Com}} i)_{k}=+1$, and also since $S \in \mathcal{W}_{k}$, and from (21), $\left\{j \in N \mid i_{j}=+1\right\} \notin \mathcal{W}_{k}$. Hence $(\overline{\operatorname{Com}} i)_{k}=-1$ and $(\overline{\operatorname{Com}} i)_{k}=+1$, a contradiction.

Consequently, by virtue of (12) and (31), the kernel of $\overline{\text { Com }}$ is given by:

$$
\mathcal{K}(\overline{\mathrm{Com}})=\left\{S \in 2^{N} \mid \omega(S) \neq \emptyset \text {, and } S^{\prime} \subset S \Rightarrow \omega\left(S^{\prime}\right)=\emptyset\right\} .
$$

Remark 3 By virtue of (31), for each set of command games $\left\{\left(N, \mathcal{W}_{k}\right) \mid k \in N\right\}$, the command influence function $\overline{\mathrm{Com}} \in \mathcal{B}$ defined in (30) is equivalent to this set of command games.

Proposition 7 Let $\left\{\left(N, \mathcal{W}_{j}\right) \mid j \in N\right\}$ be a set of command games, and $\overline{\mathrm{Com}}$ be the command influence function defined in (30). Then for each $j, k \in N$

$$
S h_{k}\left(N, \mathcal{W}_{j}\right)=\psi_{\widetilde{\alpha}^{(S h)}, \lambda=i_{k}}(\overline{\operatorname{Com}}, k \rightarrow j)-\psi_{\widehat{\alpha}^{(S h)}, \lambda=-i_{k}}(\overline{\operatorname{Com}}, k \rightarrow j)
$$

where $S h_{k}\left(N, \mathcal{W}_{j}\right)$ is the Shapley-Shubik index of player $k$ in the command game for $j$, $\psi_{\alpha, \lambda}$ is the weighted influence index defined in (13), and for each $i \in I$

$$
\widetilde{\alpha}_{i}^{(S h) k \rightarrow j}=\left\{\begin{array}{ll}
\frac{1}{n\left(\begin{array}{c}
n-1 \\
|i|-1
\end{array}\right)}, & \text { if } i_{k}=+1 \\
0, & \text { if } i_{k}=-1
\end{array} \quad \widehat{\alpha}_{i}^{(S h) k \rightarrow j}= \begin{cases}0, & \text { if } i_{k}=+1 \\
\frac{1}{n\left(\begin{array}{c}
n-1 \\
n-|i|-1
\end{array}\right)}, & \text { if } i_{k}=-1\end{cases}\right.
$$

and $|i|:=\left|\left\{m \in N \mid i_{m}=+1\right\}\right|$.

Proof: We introduce a bijection $f: I \rightarrow 2^{N}$ such that for each $i \in I$, $f(i)=\left\{k \in N \mid i_{k}=+1\right\}$. Hence, $k \in S$ iff $i_{k}=+1$. Given $\left(N, \mathcal{W}_{j}\right)$, we have by (30): $v(S)=1$ iff $S=f(i) \in \mathcal{W}_{j}$ iff $(\overline{\operatorname{Com}} i)_{j}=+1$. Hence, from (1), we have

$$
\begin{aligned}
S h_{k}\left(N, \mathcal{W}_{j}\right) & =\sum_{S \subseteq N: k \in S} \frac{(n-s) !(s-1) !}{n !}(v(S)-v(S \backslash k)) \\
& =\sum_{S \subseteq N: k \in S} \frac{(n-s) !(s-1) !}{n !} v(S)-\sum_{S \subseteq N: k \notin S} \frac{(n-s-1) ! s !}{n !} v(S) \\
& =\sum_{S \subseteq N: k \in S} \frac{v(S)}{n\left(\begin{array}{c}
n-1 \\
s-1
\end{array}\right)}-\sum_{S \subseteq N: k \notin S} \frac{v(S)}{n\left(\begin{array}{c}
n-1 \\
n-s-1
\end{array}\right)}=\sum_{i \in I_{k}^{+}} \widetilde{\alpha}_{i}^{(S h) k \rightarrow j}-\sum_{i \in I_{k}^{-}} \widehat{\alpha}_{i}^{(S h) k \rightarrow j}
\end{aligned}
$$

where

$$
I_{k}^{+}:=\left\{i \in I \mid i_{k}=+1 \wedge(\overline{\operatorname{Com}} i)_{j}=+1\right\}, \quad I_{k}^{-}:=\left\{i \in I \mid i_{k}=-1 \wedge(\overline{\operatorname{Com}} i)_{j}=+1\right\} .
$$


We have:

$$
\begin{gathered}
\sum_{i: i_{k}=+1} \widetilde{\alpha}_{i}^{(S h) k \rightarrow j}=\sum_{i: i_{k}=+1} \frac{1}{n\left(\begin{array}{c}
n-1 \\
|i|-1
\end{array}\right)}=\sum_{|i|=1}^{n} \frac{1}{n\left(\begin{array}{c}
n-1 \\
|i|-1
\end{array}\right)}\left(\begin{array}{c}
n-1 \\
|i|-1
\end{array}\right)=1 \\
\sum_{i: i_{k}=-1} \widehat{\alpha}_{i}^{(S h) k \rightarrow j}=\sum_{i: i_{k}=-1} \frac{1}{n\left(\begin{array}{c}
n-1 \\
n-|i|-1
\end{array}\right)}=\sum_{|i|=0}^{n-1} \frac{1}{n\left(\begin{array}{c}
n-1 \\
n-|i|-1
\end{array}\right)}\left(\begin{array}{c}
n-1 \\
|i|
\end{array}\right)=1,
\end{gathered}
$$

and therefore

$$
\begin{aligned}
& S h_{k}\left(N, \mathcal{W}_{j}\right)=\sum_{i \in I_{k}^{+}} \widetilde{\alpha}_{i}^{(S h) k \rightarrow j}-\sum_{i \in I_{k}^{-}} \widehat{\alpha}_{i}^{(S h) k \rightarrow j} \\
& =\frac{\sum_{i \in I_{k}^{+}} \widetilde{\alpha}_{i}^{(S h) k \rightarrow j}}{\sum_{i: i_{k}=+1} \widetilde{\alpha}_{i}^{(S h) k \rightarrow j}}-\frac{\sum_{i \in I_{k}^{-}} \widehat{\alpha}_{i}^{(S h) k \rightarrow j}}{\sum_{i: i_{k}=-1} \widehat{\alpha}_{i}^{(S h) k \rightarrow j}} \\
& =\frac{\sum_{i:(\overline{\operatorname{Com}} i)_{j}=i_{k}} \widetilde{\alpha}_{i}^{(S h) k \rightarrow j}}{\sum_{i \in I_{S}} \widetilde{\alpha}_{i}^{(S h) k \rightarrow j}}-\frac{\sum_{i:(\overline{\operatorname{Com}} i)_{j}=-i_{k}} \widehat{\alpha}_{i}^{(S h) k \rightarrow j}}{\sum_{i \in I_{S}} \widehat{\alpha}_{i}^{(S h) k \rightarrow j}} \\
& =\psi_{\widetilde{\alpha}^{(S h)}, \lambda=i_{k}}(\overline{\mathrm{Com}}, k \rightarrow j)-\psi_{\widehat{\alpha}^{(S h)}, \lambda=-i_{k}}(\overline{\mathrm{Com}}, k \rightarrow j)
\end{aligned}
$$

where $\psi_{\alpha, \lambda}$ is defined in (13), and $\widetilde{\alpha}^{(S h)}$ and $\widehat{\alpha}^{(S h)}$ are given in (33).

Proposition 8 Let $\left\{\left(N, \mathcal{W}_{j}\right) \mid j \in N\right\}$ be a set of command games, and $\overline{\mathrm{Com}}$ be the command influence function defined in (30). Then for each $j, k \in N$

$$
B z_{k}\left(N, \mathcal{W}_{j}\right)=\psi_{\widetilde{\alpha}^{(B z)}, \lambda=i_{k}}(\overline{\operatorname{Com}}, k \rightarrow j)-\psi_{\widehat{\alpha}^{(B z)}, \lambda=-i_{k}}(\overline{\operatorname{Com}}, k \rightarrow j)
$$

where $B z_{k}\left(N, \mathcal{W}_{j}\right)$ is the Banzhaf index of player $k$ in the command game for $j, \psi_{\alpha, \lambda}$ is the weighted influence index defined in (13), and for each $i \in I$

$$
\widetilde{\alpha}_{i}^{(B z) k \rightarrow j}=\left\{\begin{array}{ll}
1, & \text { if } i_{k}=+1 \\
0, & \text { if } i_{k}=-1
\end{array} \quad \widehat{\alpha}_{i}^{(B z) k \rightarrow j}= \begin{cases}0, & \text { if } i_{k}=+1 \\
1, & \text { if } i_{k}=-1 .\end{cases}\right.
$$

Proof: The proof is similar to the one of Proposition 7. From (2), we have

$$
\begin{aligned}
B z_{k}\left(N, \mathcal{W}_{j}\right) & =\frac{1}{2^{n-1}} \sum_{S \subseteq N: k \in S}(v(S)-v(S \backslash k))=\frac{1}{2^{n-1}} \sum_{S \subseteq N: k \in S} v(S)-\frac{1}{2^{n-1}} \sum_{S \subseteq N: k \notin S} v(S) \\
& =\frac{\left|\left\{i \in I \mid i_{k}=+1 \wedge(\overline{\operatorname{Com}})_{j}=+1\right\}\right|}{2^{n-1}}-\frac{\left|\left\{i \in I \mid i_{k}=-1 \wedge(\overline{\operatorname{Com}} i)_{j}=+1\right\}\right|}{2^{n-1}} \\
& =\frac{\left|\left\{i \in I \mid i_{k}=+1 \wedge(\overline{\operatorname{Com}})_{j}=+1\right\}\right|}{\left|\left\{i \in I \mid i_{k}=+1\right\}\right|}-\frac{\left|\left\{i \in I \mid i_{k}=-1 \wedge(\overline{\operatorname{Com}} i)_{j}=+1\right\}\right|}{\left|\left\{i \in I \mid i_{k}=-1\right\}\right|} \\
& =\psi_{\widetilde{\alpha}^{(B z)}, \lambda=i_{k}}(\overline{\operatorname{Com}}, k \rightarrow j)-\psi_{\widehat{\alpha}^{(B z)}, \lambda=-i_{k}}(\overline{\operatorname{Com}}, k \rightarrow j)
\end{aligned}
$$

where $\psi_{\alpha, \lambda}$ is defined in (13), and $\widetilde{\alpha}^{(B z)}$ and $\widehat{\alpha}^{(B z)}$ are given in (35). 
As in the case of the command influence function with abstention, we ask the same question about the meaning of Propositions 7 and 8. As before, under a certain assumption, both the Shapley-Shubik index and the Banzhaf index of player $k$ in the command game for $j$ are equal to the difference between the weighted influence index in which player $j$ is said to be influenced by $k$ if he follows $k$, and the weighted influence index in which the influence of $k$ on $j$ means that player $j$ 's decision is opposite to the inclination of $k$. Both weighted influence indices are measured now under the command influence function defined in (30). Moreover, the weights are different from the ones defined for the command influence function with abstention, although closely related. Remark that $\widetilde{\alpha}_{i}^{(S h) k \rightarrow j}$ and $\alpha_{i}^{(S h) k \rightarrow j}$ are identical when $i_{k}=+1$, and the former is 0 if $i_{k}=-1$. It means that for positive influence, situations where $k$ votes 'no' are discarded, which is in accordance with the definition of $\overline{\mathrm{Com}}$ (player $j$ follows the winning coalition only if this coalition votes 'yes'). The situation for $\widehat{\alpha}_{i}^{(S h) k \rightarrow j}$ is dual. The same comment applies to the case of the Banzhaf index.

\section{Command games defined for some influence functions}

In this section, we work in the opposite direction than in Section 4, and for some influence functions defined in [10] we construct the equivalent command games. In particular, we show that not for all influence functions the compatible command games exist. For each set of the equivalent command games, apart from defining the winning coalitions, we describe also the boss sets and the approval sets.

Proposition 9 Let $n \geq t>\left\lfloor\frac{n}{2}\right\rfloor$ and $\mathrm{Maj}^{[t]} \in \mathcal{B}$ be the majority function as defined in (14). Let $\left\{\left(N, \mathcal{W}_{k}^{\mathrm{Maj}^{[t]}}\right) \mid k \in N\right\}$ be a set of command games given by

$$
\mathcal{W}_{k}^{\mathrm{Maj}^{[t]}}=\{S \subseteq N \mid s \geq t\}, \forall k \in N .
$$

The majority function $\mathrm{Maj}^{[t]}$ is equivalent to the set of games $\left\{\left(N, \mathcal{W}_{k}^{\mathrm{Maj}^{[t]}}\right) \mid k \in N\right\}$.

According to Proposition 9, the command games, in which winning coalitions for each player are the ones with the cardinality at least $t, n \geq t>\left\lfloor\frac{n}{2}\right\rfloor$, are equivalent to Maj ${ }^{[t]}$.

Proof: By virtue of (36), for each $S \subseteq N$, the command function $\omega^{\mathrm{Maj}^{[t]}}(S)$ is given by

$$
\omega^{\mathrm{Maj}^{[t]}}(S)= \begin{cases}N, & \text { if } s \geq t \\ \emptyset, & \text { if } s<t\end{cases}
$$

which from $(15)$ is equal to $F_{\text {Maj }}[t](S)$.

Remark 4 Note that for the set of command games $\left\{\left(N, \mathcal{W}_{k}^{\mathrm{Maj}^{[t]}}\right) \mid k \in N\right\}$ defined by (36), we have for $n>2, n \geq t>\left\lfloor\frac{n}{2}\right\rfloor$, and $k \in N$

$$
\begin{gathered}
\operatorname{Boss}_{k}^{\mathrm{Maj}[t]}=\{S \subseteq N \mid s \geq t \wedge k \notin S\} \\
A p p_{k}^{\mathrm{Maj}^{[t]}}=\{S \subseteq N \mid s=t-1 \wedge k \notin S\} .
\end{gathered}
$$

In particular, for $t=n, k \in N$,

$$
\operatorname{Boss}_{k}^{\mathrm{Maj}}{ }^{[t]}=\emptyset, \quad A p p_{k}^{\mathrm{Maj}^{[t]}}=N \backslash k .
$$


Proposition 10 Let Gur ${ }^{[\widetilde{k}]} \in \mathcal{B}$ be the guru function as defined in (16), with the guru $\widetilde{k} \in N$. Let $\left\{\left(N, \mathcal{W}_{k}^{\text {Gur }}{ }^{[\tilde{k}]}\right) \mid k \in N\right\}$ be a set of command games given by

$$
\mathcal{W}_{k}^{\text {Gur }}{ }^{[\tilde{k}]}=\{S \subseteq N \mid \widetilde{k} \in S\}, \forall k \in N
$$

The guru function Gur ${ }^{[\widetilde{k}]}$ is equivalent to the set of games $\left\{\left(N, \mathcal{W}_{k}^{\mathrm{Gur}}{ }^{[\widetilde{k}]}\right) \mid k \in N\right\}$.

According to Proposition 10, the command games, in which winning coalitions for each player are the coalitions containing a certain player $\widetilde{k}$, are equivalent to the guru function Gur $^{[\widetilde{k}]}$ with the guru $\widetilde{k}$.

Proof: By virtue of (37), for each $S \subseteq N$, the command function $\omega^{\text {Gur }}{ }^{[\widetilde{k}]}(S)$ is given by

$$
\omega^{\text {Gur }}{ }^{[\tilde{k}]}(S)=\left\{\begin{array}{lll}
N, & \text { if } & \widetilde{k} \in S \\
\emptyset, & \text { if } \tilde{k} \notin S
\end{array}\right.
$$

which from $(17)$ is equal to $F_{\text {Gur }[\widetilde{k}]}(S)$.

Remark 5 In the command games $\left\{\left(N, \mathcal{W}_{k}^{\text {Gur }}{ }^{[\tilde{k}]}\right) \mid k \in N\right\}$ defined by (37), the guru $\widetilde{k}$ is a free agent, and the remaining players are cogs, i.e.,

$$
\begin{gathered}
\operatorname{Boss}_{\widetilde{k}}^{\operatorname{Gur}^{[\widetilde{k}]}}=\emptyset, \quad \operatorname{App}_{\widetilde{k}}^{\text {Gur }^{[\widetilde{k}]}}=2^{N \backslash \widetilde{k}} \\
\operatorname{Boss}_{k}^{\text {Gur }^{[\widetilde{k}]}}=\{S \subseteq N \mid \widetilde{k} \in S \wedge k \notin S\}, \quad \operatorname{App}_{k}^{\text {Gur }^{[\widetilde{k}]}}=\emptyset, \quad \text { for } k \neq \widetilde{k} .
\end{gathered}
$$

Proposition 11 Let $\mathrm{Id} \in \mathcal{B}$ be the identity function as defined in (18). Let $\left\{\left(N, \mathcal{W}_{k}^{\text {ld }}\right) \mid k \in N\right\}$ be a set of command games given by

$$
\mathcal{W}_{k}^{\text {ld }}=\{S \subseteq N \mid k \in S\}, \forall k \in N .
$$

The identity function Id is equivalent to the set of games $\left\{\left(N, \mathcal{W}_{k}^{\text {ld }}\right) \mid k \in N\right\}$.

According to Proposition 11, the command games in which, for each player $k$, winning coalitions for $k$ are the coalitions containing $k$, are equivalent to the identity function.

Proof: ¿From (38), we have $\omega^{\text {ld }}(S)=S$ for each $S \subseteq N$. On the other hand, also $F_{\text {ld }}(S)=S$ for $S \subseteq N$.

Remark 6 In the command games $\left\{\left(N, \mathcal{W}_{k}^{\text {ld }}\right) \mid k \in N\right\}$ defined by (38), all players are free agents, i.e.,

$$
\operatorname{Boss}_{k}^{\text {ld }}=\emptyset, \quad A p p_{k}^{\text {ld }}=2^{N \backslash k} .
$$

Proposition 12 Let $-\mathrm{Id} \in \mathcal{B}$ be the reversal function as defined in (19). There is no set of command games such that the reversal function is compatible with these command games.

Proof: We know that $F_{-\mathrm{ld}}(S)=\emptyset$ for $S \subseteq N$. Hence, in particular, $F_{-\mathrm{ld}}(N)=\emptyset$. But for any set of command games, we have $\omega(N)=N$, and therefore there is no command game compatible with - Id. 


\section{Example - The Confucian model}

In [14] the Confucian model of society is mentioned. We have four players in the society, i.e., $N=\{1,2,3,4\}$, with the king (1), the man (2), the wife (3), and the child (4). The rules are as follows:

(i) The man follows the king;

(ii) The wife and the child follow the man;

(iii) The king should respect his people.

Let us define the command games for this example. By virtue of the rules (i) and (ii), we have immediately:

$$
\begin{aligned}
& \mathcal{W}_{2}=\{1,12,13,14,123,124,134,1234\} \\
& \mathcal{W}_{3}=\{2,12,23,24,123,124,234,1234\} \\
& \mathcal{W}_{4}=\{2,12,23,24,123,124,234,1234\}
\end{aligned}
$$

Hence, we have

$$
\begin{gathered}
\text { Boss }_{2}=\{1,13,14,134\}, \quad \text { Boss }_{3}=\{2,12,24,124\}, \quad \text { Boss }_{4}=\{2,12,23,123\} \\
\text { App }_{2}=\text { App }_{3}=A p p_{4}=\emptyset
\end{gathered}
$$

which means that players 2,3 , and 4 are cogs.

How can we translate the rule (iii) into the set $\mathcal{W}_{1}$ of winning coalitions in the command game for player 1? We propose several interpretations of this rule, and consequently, several command games for player 1 .

\subsection{The command game with $\mathcal{W}_{1}=\{1234\}$}

If $\mathcal{W}_{1}=\{1234\}$, then we get:

$$
\text { Boss }_{1}=\emptyset, \quad A p p_{1}=\{234\},
$$

i.e., the king needs the approval of all his people.

$$
\begin{gathered}
\omega(1)=\omega(13)=\omega(14)=\omega(134)=\{2\}, \quad \omega(2)=\omega(23)=\omega(24)=\omega(234)=\{3,4\} \\
\omega(3)=\omega(4)=\omega(34)=\emptyset, \quad \omega(N)=N, \quad \omega(12)=\omega(123)=\omega(124)=\{2,3,4\} .
\end{gathered}
$$

The Shapley-Shubik index matrix is then:

$$
P=\left[S h_{k}\left(N, \mathcal{W}_{j}\right)\right]_{j, k=1}^{n}=\left[\begin{array}{cccc}
\frac{1}{4} & \frac{1}{4} & \frac{1}{4} & \frac{1}{4} \\
1 & 0 & 0 & 0 \\
0 & 1 & 0 & 0 \\
0 & 1 & 0 & 0
\end{array}\right]
$$

and from (9) one has

$$
\left\{\begin{array}{c}
\pi_{1}=\frac{1}{4} \pi_{1}+\pi_{2} \\
\pi_{2}=\frac{1}{4} \pi_{1}+\pi_{3}+\pi_{4} \\
\pi_{3}=\frac{1}{4} \pi_{1} \\
\pi_{4}=\frac{1}{4} \pi_{1} \\
\pi_{1}+\pi_{2}+\pi_{3}+\pi_{4}=1
\end{array}\right.
$$


which gives the authority distribution:

$$
\pi=\frac{1}{9}(4,3,1,1)
$$

Let us apply now the command influence functions to the model. Table 1 presents the inclination and decision vectors under the three command influence functions.

Table 1. The inclination and decision vectors for $\mathcal{W}_{1}=\{1234\}$

\begin{tabular}{c||c|c|c}
$i \in I$ & Com $i$ & $\widetilde{\operatorname{Com} i}$ & $\overline{\operatorname{Com}} i$ \\
\hline \hline$(1,1,1,1)$ & $(1,1,1,1)$ & $(1,1,1,1)$ & $(1,1,1,1)$ \\
$(1,1,1,-1)$ & $(0,1,1,1)$ & $(1,1,1,1)$ & $(-1,1,1,1)$ \\
$(1,1,-1,1)$ & $(0,1,1,1)$ & $(1,1,1,1)$ & $(-1,1,1,1)$ \\
$(1,-1,1,1)$ & $(0,1,-1,-1)$ & $(1,1,-1,-1)$ & $(-1,1,-1,-1)$ \\
$(-1,1,1,1)$ & $(0,-1,1,1)$ & $(-1,-1,1,1)$ & $(-1,-1,1,1)$ \\
$(1,-1,1,-1)$ & $(0,1,-1,-1)$ & $(1,1,-1,-1)$ & $(-1,1,-1,-1)$ \\
$(1,1,-1,-1)$ & $(0,1,1,1)$ & $(1,1,1,1)$ & $(-1,1,1,1)$ \\
$(-1,1,1,-1)$ & $(0,-1,1,1)$ & $(-1,-1,1,1)$ & $(-1,-1,1,1)$ \\
$(1,-1,-1,1)$ & $(0,1,-1,-1)$ & $(1,1,-1,-1)$ & $(-1,1,-1,-1)$ \\
$(-1,-1,1,1)$ & $(0,-1,-1,-1)$ & $(-1,-1,-1,-1)$ & $(-1,-1,-1,-1)$ \\
$(-1,1,-1,1)$ & $(0,-1,1,1)$ & $(-1,-1,1,1)$ & $(-1,-1,1,1)$ \\
$(1,-1,-1,-1)$ & $(0,1,-1,-1)$ & $(1,1,-1,-1)$ & $(-1,1,-1,-1)$ \\
$(-1,1,-1,-1)$ & $(0,-1,1,1)$ & $(-1,-1,1,1)$ & $(-1,-1,1,1)$ \\
$(-1,-1,1,-1)$ & $(0,-1,-1,-1)$ & $(-1,-1,-1,-1)$ & $(-1,-1,-1,-1)$ \\
$(-1,-1,-1,1)$ & $(0,-1,-1,-1)$ & $(-1,-1,-1,-1)$ & $(-1,-1,-1,-1)$ \\
$(-1,-1,-1,-1)$ & $(-1,-1,-1,-1)$ & $(-1,-1,-1,-1)$ & $(-1,-1,-1,-1)$
\end{tabular}

Note that under $B \in\{\mathrm{Com}, \widetilde{\mathrm{Com}}, \overline{\mathrm{Com}}\}$ each player (except the king) always follows the inclination of his boss sets. Under the influence function Com, the king will say 'yes' / 'no' only if all players (including himself) have the positive / negative inclination. In the remaining cases, the king abstains. Under the influence function $\widetilde{\mathrm{Com}}$, the king always follows his own inclination, since 1234 is the unique winning coalition in the command game for player 1 . Under the influence function $\overline{\mathrm{Com}}$, if all people of the king are against, his decision is also 'no', but if all his people are in favor (i.e., the king has the approval of his people), his decision is 'yes' only if his inclination is also positive. In case the people of the king are not unanimous, the king has no approval, and consequently he chooses 'no', even if his inclination is positive.

We have, in particular:

$$
\begin{gathered}
F_{B}(1)=\{2\}, \quad F_{B}(2)=\{3,4\}, \quad F_{B}(12)=\{2,3,4\}, \quad \text { for } B \in\{\mathrm{Com}, \overline{\mathrm{Com}}\} \\
F_{\widetilde{\mathrm{Com}}}(1)=\{1,2\}, \quad F_{\widetilde{\mathrm{Com}}}(2)=\{3,4\}, \quad F_{\widetilde{\mathrm{Com}}}(12)=N .
\end{gathered}
$$

We have, $d_{\alpha}(B, S \rightarrow j)=1$ for each $j \in F_{B}(S) \backslash S$. Hence, we have:

$$
d_{\alpha}(B, 1 \rightarrow 2)=d_{\alpha}(B, 2 \rightarrow 3)=d_{\alpha}(B, 2 \rightarrow 4)=1, \quad \text { for } B \in\{\operatorname{Com}, \widetilde{\operatorname{Com}}, \overline{\operatorname{Com}}\}
$$

The kernel is equal to:

$$
\mathcal{K}(B)=\{\{1\},\{2\}\}, \quad \text { for } B \in\{\operatorname{Com}, \widetilde{\operatorname{Com}}, \overline{\operatorname{Com}}\} .
$$


We have already calculated the Shapley-Shubik index matrix for this example. The Banzhaf index matrices are equal to:

$$
B z=\left[B z_{k}\left(N, \mathcal{W}_{j}\right)\right]_{j, k=1}^{n}=\left[\begin{array}{cccc}
\frac{1}{8} & \frac{1}{8} & \frac{1}{8} & \frac{1}{8} \\
1 & 0 & 0 & 0 \\
0 & 1 & 0 & 0 \\
0 & 1 & 0 & 0
\end{array}\right] \quad \widetilde{B z}=\left[\widetilde{B z}_{k}\left(N, \mathcal{W}_{j}\right)\right]_{j, k=1}^{n}=\left[\begin{array}{cccc}
\frac{1}{4} & \frac{1}{4} & \frac{1}{4} & \frac{1}{4} \\
1 & 0 & 0 & 0 \\
0 & 1 & 0 & 0 \\
0 & 1 & 0 & 0
\end{array}\right]
$$

\subsection{The command game with $\mathcal{W}_{1}=\{123,124,134,1234\}$}

If $\mathcal{W}_{1}=\{123,124,134,1234\}$, then we get:

$$
\text { Boss }_{1}=\emptyset, \quad A p p_{1}=\{23,24,34,234\},
$$

i.e., the king needs the approval of the majority of his people.

$$
\begin{gathered}
\omega(1)=\omega(13)=\omega(14)=\{2\}, \quad \omega(134)=\{1,2\}, \quad \omega(2)=\omega(23)=\omega(24)=\omega(234)=\{3,4\} \\
\omega(3)=\omega(4)=\omega(34)=\emptyset, \quad \omega(12)=\{2,3,4\}, \quad \omega(123)=\omega(124)=\omega(N)=N .
\end{gathered}
$$

The Shapley-Shubik index matrix is then:

$$
P=\left[S h_{k}\left(N, \mathcal{W}_{j}\right)\right]_{j, k=1}^{n}=\left[\begin{array}{cccc}
\frac{1}{2} & \frac{1}{6} & \frac{1}{6} & \frac{1}{6} \\
1 & 0 & 0 & 0 \\
0 & 1 & 0 & 0 \\
0 & 1 & 0 & 0
\end{array}\right]
$$

and from (9) one has

$$
\left\{\begin{array}{c}
\pi_{1}=\frac{1}{2} \pi_{1}+\pi_{2} \\
\pi_{2}=\frac{1}{6} \pi_{1}+\pi_{3}+\pi_{4} \\
\pi_{3}=\frac{1}{6} \pi_{1} \\
\pi_{4}=\frac{1}{6} \pi_{1} \\
\pi_{1}+\pi_{2}+\pi_{3}+\pi_{4}=1
\end{array}\right.
$$

which gives the authority distribution:

$$
\pi=\frac{1}{11}(6,3,1,1)
$$

As before, we apply now the command influence functions to the model. Table 2 presents the inclination and decision vectors under the three command influence functions.

Note that for $\mathcal{W}_{1}=\{123,124,134,1234\}$ the decision vectors under $\widetilde{\text { Com }}$ are the same as the decision vectors under $\widetilde{\text { Com }}$ for $\mathcal{W}_{1}=\{1234\}$.

The results are similar as before, that is:

$$
\begin{gathered}
F_{B}(1)=\{2\}, \quad F_{B}(2)=\{3,4\}, \quad F_{B}(12)=\{2,3,4\}, \quad \text { for } B \in\{\mathrm{Com}, \overline{\operatorname{Com}}\} \\
F_{\widetilde{\mathrm{Com}}}(1)=\{1,2\}, \quad F_{\widetilde{\mathrm{Com}}}(2)=\{3,4\}, \quad F_{\widetilde{\mathrm{Com}}}(12)=N .
\end{gathered}
$$

In particular:

$$
d_{\alpha}(B, 1 \rightarrow 2)=d_{\alpha}(B, 2 \rightarrow 3)=d_{\alpha}(B, 2 \rightarrow 4)=1, \quad \text { for } B \in\{\operatorname{Com}, \widetilde{\operatorname{Com}}, \overline{\operatorname{Com}}\}
$$


Table 2. The inclination and decision vectors for $\mathcal{W}_{1}=\{123,124,134,1234\}$

\begin{tabular}{c||c|c|c}
$i \in I$ & Comi & $\widetilde{\operatorname{Com} i}$ & $\overline{\text { Com } i}$ \\
\hline \hline$(1,1,1,1)$ & $(1,1,1,1)$ & $(1,1,1,1)$ & $(1,1,1,1)$ \\
$(1,1,1,-1)$ & $(1,1,1,1)$ & $(1,1,1,1)$ & $(1,1,1,1)$ \\
$(1,1,-1,1)$ & $(1,1,1,1)$ & $(1,1,1,1)$ & $(1,1,1,1)$ \\
$(1,-1,1,1)$ & $(1,1,-1,-1)$ & $(1,1,-1,-1)$ & $(1,1,-1,-1)$ \\
$(-1,1,1,1)$ & $(0,-1,1,1)$ & $(-1,-1,1,1)$ & $(-1,-1,1,1)$ \\
$(1,-1,1,-1)$ & $(0,1,-1,-1)$ & $(1,1,-1,-1)$ & $(-1,1,-1,-1)$ \\
$(1,1,-1,-1)$ & $(0,1,1,1)$ & $(1,1,1,1)$ & $(-1,1,1,1)$ \\
$(-1,1,1,-1)$ & $(0,-1,1,1)$ & $(-1,-1,1,1)$ & $(-1,-1,1,1)$ \\
$(1,-1,-1,1)$ & $(0,1,-1,-1)$ & $(1,1,-1,-1)$ & $(-1,1,-1,-1)$ \\
$(-1,-1,1,1)$ & $(0,-1,-1,-1)$ & $(-1,-1,-1,-1)$ & $(-1,-1,-1,-1)$ \\
$(-1,1,-1,1)$ & $(0,-1,1,1)$ & $(-1,-1,1,1)$ & $(-1,-1,1,1)$ \\
$(1,-1,-1,-1)$ & $(0,1,-1,-1)$ & $(1,1,-1,-1)$ & $(-1,1,-1,-1)$ \\
$(-1,1,-1,-1)$ & $(-1,-1,1,1)$ & $(-1,-1,1,1)$ & $(-1,-1,1,1)$ \\
$(-1,-1,1,-1)$ & $(-1,-1,-1,-1)$ & $(-1,-1,-1,-1)$ & $(-1,-1,-1,-1)$ \\
$(-1,-1,-1,1)$ & $(-1,-1,-1,-1)$ & $(-1,-1,-1,-1)$ & $(-1,-1,-1,-1)$ \\
$(-1,-1,-1,-1)$ & $(-1,-1,-1,-1)$ & $(-1,-1,-1,-1)$ & $(-1,-1,-1,-1)$
\end{tabular}

The kernel is equal to:

$$
\mathcal{K}(B)=\{\{1\},\{2\}\}, \quad \text { for } B \in\{\operatorname{Com}, \widetilde{\operatorname{Com}}, \overline{\operatorname{Com}}\} .
$$

The Banzhaf index matrices are equal to:

$$
B z=\left[B z_{k}\left(N, \mathcal{W}_{j}\right)\right]_{j, k=1}^{n}=\left[\begin{array}{cccc}
\frac{1}{2} & \frac{1}{4} & \frac{1}{4} & \frac{1}{4} \\
1 & 0 & 0 & 0 \\
0 & 1 & 0 & 0 \\
0 & 1 & 0 & 0
\end{array}\right] \quad \widetilde{B z}=\left[\widetilde{B z} z_{k}\left(N, \mathcal{W}_{j}\right)\right]_{j, k=1}^{n}=\left[\begin{array}{cccc}
\frac{2}{5} & \frac{1}{5} & \frac{1}{5} & \frac{1}{5} \\
1 & 0 & 0 & 0 \\
0 & 1 & 0 & 0 \\
0 & 1 & 0 & 0
\end{array}\right]
$$

\subsection{The command game with $\mathcal{W}_{1}=\{12,13,14,123,124,134,1234\}$}

If $\mathcal{W}_{1}=\{12,13,14,123,124,134,1234\}$, then we get:

$$
\text { Boss }_{1}=\emptyset, \quad A p p_{1}=\{2,3,4,23,24,34,234\},
$$

i.e., the king needs the approval of at least one of his people.

$$
\begin{gathered}
\omega(1)=\{2\}, \quad \omega(13)=\omega(14)=\omega(134)=\{1,2\}, \quad \omega(2)=\omega(23)=\omega(24)=\omega(234)=\{3,4\} \\
\omega(3)=\omega(4)=\omega(34)=\emptyset, \quad \omega(12)=\omega(123)=\omega(124)=\omega(N)=N .
\end{gathered}
$$

The Shapley-Shubik index matrix is then:

$$
P=\left[S h_{k}\left(N, \mathcal{W}_{j}\right)\right]_{j, k=1}^{n}=\left[\begin{array}{cccc}
\frac{3}{4} & \frac{1}{12} & \frac{1}{12} & \frac{1}{12} \\
1 & 0 & 0 & 0 \\
0 & 1 & 0 & 0 \\
0 & 1 & 0 & 0
\end{array}\right]
$$

and from (9) one has

$$
\left\{\begin{array}{c}
\pi_{1}=\frac{3}{4} \pi_{1}+\pi_{2} \\
\pi_{2}=\frac{1}{12} \pi_{1}+\pi_{3}+\pi_{4} \\
\pi_{3}=\frac{1}{12} \pi_{1} \\
\pi_{4}=\frac{1}{12} \pi_{1} \\
\pi_{1}+\pi_{2}+\pi_{3}+\pi_{4}=1
\end{array}\right.
$$


which gives the authority distribution:

$$
\pi=\frac{1}{17}(12,3,1,1)
$$

We apply the command influence functions to the model. Table 3 presents the inclination and decision vectors under the command influence functions.

Table 3. The inclination and decision vectors for $\mathcal{W}_{1}=\{12,13,14,123,124,134,1234\}$

\begin{tabular}{c||c|c|c}
$i \in I$ & Comi & $\widetilde{\text { Com } i}$ & $\overline{\text { Com } i}$ \\
\hline \hline$(1,1,1,1)$ & $(1,1,1,1)$ & $(1,1,1,1)$ & $(1,1,1,1)$ \\
$(1,1,1,-1)$ & $(1,1,1,1)$ & $(1,1,1,1)$ & $(1,1,1,1)$ \\
$(1,1,-1,1)$ & $(1,1,1,1)$ & $(1,1,1,1)$ & $(1,1,1,1)$ \\
$(1,-1,1,1)$ & $(1,1,-1,-1)$ & $(1,1,-1,-1)$ & $(1,1,-1,-1)$ \\
$(-1,1,1,1)$ & $(0,-1,1,1)$ & $(-1,-1,1,1)$ & $(-1,-1,1,1)$ \\
$(1,-1,1,-1)$ & $(1,1,-1,-1)$ & $(1,1,-1,-1)$ & $(1,1,-1,-1)$ \\
$(1,1,-1,-1)$ & $(1,1,1,1)$ & $(1,1,1,1)$ & $(1,1,1,1)$ \\
$(-1,1,1,-1)$ & $(-1,-1,1,1)$ & $(-1,-1,1,1)$ & $(-1,-1,1,1)$ \\
$(1,-1,-1,1)$ & $(1,1,-1,-1)$ & $(1,1,-1,-1)$ & $(1,1,-1,-1)$ \\
$(-1,-1,1,1)$ & $(-1,-1,-1,-1)$ & $(-1,-1,-1,-1)$ & $(-1,-1,-1,-1)$ \\
$(-1,1,-1,1)$ & $(-1,-1,1,1)$ & $(-1,-1,1,1)$ & $(-1,-1,1,1)$ \\
$(1,-1,-1,-1)$ & $(0,1,-1,-1)$ & $(1,1,-1,-1)$ & $(-1,1,-1,-1)$ \\
$(-1,1,-1,-1)$ & $(-1,-1,1,1)$ & $(-1,-1,1,1)$ & $(-1,-1,1,1)$ \\
$(-1,-1,1,-1)$ & $(-1,-1,-1,-1)$ & $(-1,-1,-1,-1)$ & $(-1,-1,-1,-1)$ \\
$(-1,-1,-1,1)$ & $(-1,-1,-1,-1)$ & $(-1,-1,-1,-1)$ & $(-1,-1,-1,-1)$ \\
$(-1,-1,-1,-1)$ & $(-1,-1,-1,-1)$ & $(-1,-1,-1,-1)$ & $(-1,-1,-1,-1)$
\end{tabular}

As before, under all three influence functions, each player (except the king) always follows the inclination of his boss sets. Moreover, under Com, the king almost always follows his inclination, except two cases of abstention when the inclination of all his people is

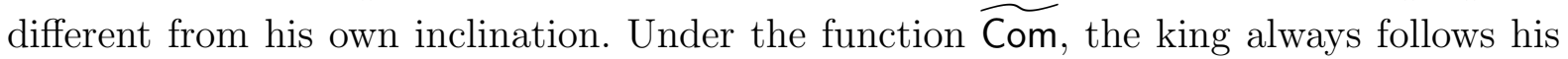
own inclination as in the previous two command games for player 1. Under the function $\overline{\mathrm{Com}}$, the king almost always follows his inclination, except one case when his inclination is positive while the inclination of all his people is negative.

We have also:

$$
\begin{gathered}
F_{B}(1)=\{2\}, \quad F_{B}(2)=\{3,4\}, \quad F_{B}(12)=N, \quad \text { for } B \in\{\operatorname{Com}, \overline{\operatorname{Com}}\} \\
F_{\widetilde{\mathrm{Com}}}(1)=\{1,2\}, \quad F_{\widetilde{\mathrm{Com}}}(2)=\{3,4\}, \quad F_{\widetilde{\mathrm{Com}}}(12)=N . \\
d_{\alpha}(B, 1 \rightarrow 2)=d_{\alpha}(B, 2 \rightarrow 3)=d_{\alpha}(B, 2 \rightarrow 4)=1, \quad \text { for } B \in\{\operatorname{Com}, \widetilde{\operatorname{Com}}, \overline{\operatorname{Com}}\} . \\
\mathcal{K}(B)=\{\{1\},\{2\}\}, \quad \text { for } B \in\{\operatorname{Com}, \widetilde{\mathrm{Com}}, \overline{\mathrm{Com}}\} .
\end{gathered}
$$

The Banzhaf index matrices are equal to:

$$
B z=\left[B z_{k}\left(N, \mathcal{W}_{j}\right)\right]_{j, k=1}^{n}=\left[\begin{array}{cccc}
\frac{7}{8} & \frac{1}{8} & \frac{1}{8} & \frac{1}{8} \\
1 & 0 & 0 & 0 \\
0 & 1 & 0 & 0 \\
0 & 1 & 0 & 0
\end{array}\right] \quad \widetilde{B z}=\left[\widetilde{B z}_{k}\left(N, \mathcal{W}_{j}\right)\right]_{j, k=1}^{n}=\left[\begin{array}{cccc}
\frac{7}{10} & \frac{1}{10} & \frac{1}{10} & \frac{1}{10} \\
1 & 0 & 0 & 0 \\
0 & 1 & 0 & 0 \\
0 & 1 & 0 & 0
\end{array}\right] .
$$




\section{Conclusion}

This paper presents a comparison between two models that deal with modeling players' interactions: the command games and the influence model. The link between the models is expressed by defining the influence functions that are compatible with or equivalent to the command games. An influence function and the command games are compatible if each commandable player for a coalition in the command game is a follower of the coalition under the command influence function. An influence function and the command games are equivalent if they are compatible, and additionally each follower of a coalition under the command influence function is the commandable player for that coalition in the command game. Consequently, for each set of the command games, we define two equivalent influence functions and one compatible influence function. Moreover, for some influence functions we define the equivalent command games. Nevertheless, not for all influence functions the compatible command games exist. In the paper, we also show links between some power indices, which can be used in the command games, and the generalized weighted influence indices. We note that results concerning the Shapley-Shubik index and the Banzhaf index differ only in the weights for the influence indices. The concluding remark can be that the influence model is more general than the framework of the command games, and the concepts of the influence function and the influence index can capture the command structure.

A research agenda concerning our future work on the influence model contains several issues. In particular, we plan to introduce the authority distribution based on the influence indices. Moreover, we intend to introduce dynamic aspects into the model. We want to study the behavior of the series $B i, B^{2} i, \ldots, B^{n} i, \ldots$, to find convergence conditions, to investigate the corresponding influence indices, and relations between the repeated influence model and the command games. We plan to analyze a generalized model of influence, in which each player has a continuum of options to choose (that is, a model in which the inclination or opinion of each player lies in an interval, say $[0,1]$, where each $i_{k} \in[0,1]$ can be interpreted as player $k$ 's degree of inclination to say 'yes'). An important issue for future research concerns an axiomatic characterization of the influence indices. Furthermore, it would be interesting to test the new concepts and to run lab experiments concerning the influence between players.

\section{Appendix - relations between the weighted influence indices and the power indices}

Next, we show relations between some power indices and the weighted influence indices under the command influence functions defined in Section 4.

Proposition 13 Let $j \in N$ and $\left(N, \mathcal{W}_{j}\right)$ be the command game for $j$, and Com $\in \mathcal{B}$ be the command influence function defined by (22). Then

$$
A\left(N, \mathcal{W}_{j}\right)=\frac{\psi_{\alpha, \lambda \neq 0}(\operatorname{Com}, k \rightarrow j)}{2}, \forall k \in N
$$

and for each $k \in N$

$$
\operatorname{Col}_{k}^{P}\left(N, \mathcal{W}_{j}\right)=\frac{\psi_{\alpha, \lambda=i_{k}}(\operatorname{Com}, k \rightarrow j)-\psi_{\alpha, \lambda=-i_{k}}(\operatorname{Com}, k \rightarrow j)}{\psi_{\alpha, \lambda \neq 0}(\operatorname{Com}, k \rightarrow j)}
$$




$$
K B_{k}\left(N, \mathcal{W}_{j}\right)=\frac{\psi_{\alpha, \lambda=i_{k}}(\operatorname{Com}, k \rightarrow j)}{\psi_{\alpha, \lambda \neq 0}(\operatorname{Com}, k \rightarrow j)}
$$

where $A\left(N, \mathcal{W}_{j}\right)$ is the Coleman power of a collectivity to act in the command game for player $j, \operatorname{Col}_{k}^{P}\left(N, \mathcal{W}_{j}\right)$ is the Coleman index to prevent action of player $k$ in the command game for $j$, and $K B_{k}\left(N, \mathcal{W}_{j}\right)$ is the König-Bräuninger inclusiveness index of $k$ in the command game for $j$. Moreover, $\psi_{\alpha, \lambda}$ is the weighted influence index defined in (13) with

$$
\alpha_{i}^{k \rightarrow j}=1, \forall i \in I \text {. }
$$

Proof: (39) results immediately from (3). Let $j \in N$, and take an arbitrary $k \in N$. We have

$$
A\left(N, \mathcal{W}_{j}\right)=\frac{\sum_{S \subseteq N} v(S)}{2^{n}}=\frac{\left|\left\{i \in I \mid(\operatorname{Com} i)_{j} \neq 0\right\}\right|}{2|I|}=\frac{\psi_{\alpha, \lambda \neq 0}(\operatorname{Com}, k \rightarrow j)}{2}
$$

where $\alpha$ is given in (41).

We have

$$
\sum_{S \subseteq N} v(S)=\frac{\left|\left\{i \in I \mid(\operatorname{Com} i)_{j} \neq 0\right\}\right|}{2}=2^{n-1} \psi_{\alpha, \lambda \neq 0}(\operatorname{Com}, k \rightarrow j)>0 .
$$

By virtue of (2) and (4), we have for each $j, k \in N$

$$
\operatorname{Col}_{k}^{P}\left(N, \mathcal{W}_{j}\right)=\frac{2^{n-1} B z_{k}\left(N, \mathcal{W}_{j}\right)}{\sum_{S \subseteq N} v(S)}=\frac{B z_{k}\left(N, \mathcal{W}_{j}\right)}{\psi_{\alpha, \lambda \neq 0}(\operatorname{Com}, k \rightarrow j)}
$$

which together with (26) gives (40).

Since we have

$$
\sum_{S \subseteq N: k \in S} v(S)=\frac{\left|\left\{i \in I \mid(\operatorname{Com} i)_{j}=i_{k}\right\}\right|}{2}=2^{n-1} \psi_{\alpha, \lambda=i_{k}}(\operatorname{Com}, k \rightarrow j),
$$

hence from (6), (42), and (43), we have

$$
K B_{k}\left(N, \mathcal{W}_{j}\right)=\frac{\sum_{S \subseteq N: k \in S} v(S)}{\sum_{S \subseteq N} v(S)}=\frac{\psi_{\alpha, \lambda=i_{k}}(\operatorname{Com}, k \rightarrow j)}{\psi_{\alpha, \lambda \neq 0}(\operatorname{Com}, k \rightarrow j)}
$$

Proposition 14 Let $j \in N$ and $\left(N, \mathcal{W}_{j}\right)$ be the command game for $j$, and $\overline{\operatorname{Com}} \in \mathcal{B}$ be the command influence function defined by (30). Then

$$
A\left(N, \mathcal{W}_{j}\right)=\psi_{\alpha, \lambda=+1}(\overline{\mathrm{Com}}, k \rightarrow j), \forall k \in N
$$

and for each $k \in N$

$$
\operatorname{Col}_{k}^{P}\left(N, \mathcal{W}_{j}\right)=\frac{\psi_{\widetilde{\alpha}^{(B z)}, \lambda=i_{k}}(\overline{\operatorname{Com}}, k \rightarrow j)-\psi_{\widehat{\alpha}^{(B z)}, \lambda=-i_{k}}(\overline{\operatorname{Com}}, k \rightarrow j)}{2 \psi_{\alpha, \lambda=+1}(\overline{\operatorname{Com}}, k \rightarrow j)}
$$




$$
\begin{gathered}
\operatorname{Col}_{k}^{I}\left(N, \mathcal{W}_{j}\right)=\frac{\psi_{\widetilde{\alpha}^{(B z)}, \lambda=i_{k}}(\overline{\operatorname{Com}}, k \rightarrow j)-\psi_{\widehat{\alpha}^{(B z)}, \lambda=-i_{k}}(\overline{\operatorname{Com}}, k \rightarrow j)}{2 \psi_{\alpha, \lambda=-1}(\overline{\operatorname{Com}}, k \rightarrow j)} \\
K B_{k}\left(N, \mathcal{W}_{j}\right)=\frac{\psi_{\widetilde{\alpha}^{(B z)}, \lambda=i_{k}}(\overline{\operatorname{Com}}, k \rightarrow j)}{2 \psi_{\alpha, \lambda=+1}(\overline{\operatorname{Com}}, k \rightarrow j)}
\end{gathered}
$$

where $A\left(N, \mathcal{W}_{j}\right)$ is the Coleman power of a collectivity to act in the command game for player $j, \operatorname{Col}_{k}^{P}\left(N, \mathcal{W}_{j}\right) / \operatorname{Col}_{k}^{I}\left(N, \mathcal{W}_{j}\right)$ is the Coleman index to prevent action / the Coleman index to initiate action of player $k$ in the command game for $j$, and $K B_{k}\left(N, \mathcal{W}_{j}\right)$ is the König-Bräuninger inclusiveness index of $k$ in the command game for $j$. Moreover, $\psi_{\alpha, \lambda}$ is the weighted influence index defined in (13), $\alpha$ is given in (41), and $\widetilde{\alpha}^{(B z)}, \widehat{\alpha}^{(B z)}$ are given in (35).

Proof: (44) results immediately from (3). Let $j \in N$, and take an arbitrary $k \in N$. We have

$$
A\left(N, \mathcal{W}_{j}\right)=\frac{\sum_{S \subseteq N} v(S)}{2^{n}}=\frac{\left|\left\{i \in I \mid(\overline{\operatorname{Com}} i)_{j}=+1\right\}\right|}{|I|}=\psi_{\alpha, \lambda=+1}(\overline{\operatorname{Com}}, k \rightarrow j)
$$

where $\alpha$ is given in (41).

We have

$$
\sum_{S \subseteq N} v(S)=\left|\left\{i \in I \mid(\overline{\operatorname{Com}} i)_{j}=+1\right\}\right|=2^{n} \psi_{\alpha, \lambda=+1}(\overline{\operatorname{Com}}, k \rightarrow j)>0 .
$$

By virtue of (2) and (4), we have for each $j, k \in N$

$$
\operatorname{Col}_{k}^{P}\left(N, \mathcal{W}_{j}\right)=\frac{2^{n-1} B z_{k}\left(N, \mathcal{W}_{j}\right)}{\sum_{S \subseteq N} v(S)}=\frac{B z_{k}\left(N, \mathcal{W}_{j}\right)}{2 \psi_{\alpha, \lambda=+1}(\overline{\mathrm{Com}}, k \rightarrow j)}
$$

which together with (34) gives (45).

Moreover we note that

$$
\begin{gathered}
\sum_{S \subseteq N: k \in S} v(S)=\left|\left\{i \in I \mid i_{k}=+1 \wedge(\overline{\operatorname{Com}} i)_{j}=+1\right\}\right|=2^{n-1} \psi_{\widetilde{\alpha}^{(B z)}, \lambda=i_{k}}(\overline{\operatorname{Com}}, k \rightarrow j) \\
\sum_{S \subseteq N: k \notin S} v(S)=\left|\left\{i \in I \mid i_{k}=-1 \wedge(\overline{\operatorname{Com}} i)_{j}=+1\right\}\right|=2^{n-1} \psi_{\widehat{\alpha}^{(B z)}, \lambda=-i_{k}}(\overline{\operatorname{Com}}, k \rightarrow j) \\
2^{n}-\sum_{S \subseteq N} v(S)=\left|\left\{i \in I \mid(\overline{\operatorname{Com}} i)_{j}=-1\right\}\right|=2^{n} \psi_{\alpha, \lambda=-1}(\overline{\operatorname{Com}}, k \rightarrow j)
\end{gathered}
$$

By virtue of (5), (47), (48), and (49), we have

$$
\begin{aligned}
\operatorname{Col}_{k}^{I}\left(N, \mathcal{W}_{j}\right) & =\frac{\sum_{S \subseteq N: k \notin S}(v(S \cup k)-v(S))}{2^{n}-\sum_{S \subseteq N} v(S)}=\frac{\sum_{S \subseteq N: k \in S} v(S)-\sum_{S \subseteq N: k \notin S} v(S)}{2^{n}-\sum_{S \subseteq N} v(S)} \\
& =\frac{\left|\left\{i \in I \mid i_{k}=+1 \wedge(\overline{\operatorname{Com}} i)_{j}=+1\right\}\right|-\left|\left\{i \in I \mid i_{k}=-1 \wedge(\overline{\operatorname{Com}} i)_{j}=+1\right\}\right|}{\left|\left\{i \in I \mid(\overline{\operatorname{Com}} i)_{j}=-1\right\}\right|} \\
& =\frac{\psi_{\widetilde{\alpha}(B z), \lambda=i_{k}}(\overline{\operatorname{Com}}, k \rightarrow j)-\psi_{\widehat{\alpha}(B z), \lambda=-i_{k}}(\overline{\operatorname{Com}}, k \rightarrow j)}{2 \psi_{\alpha, \lambda=-1}(\overline{\operatorname{Com}}, k \rightarrow j)}
\end{aligned}
$$


From (6), (46), and (47), we have

$$
K B_{k}\left(N, \mathcal{W}_{j}\right)=\frac{\sum_{S \subseteq N: k \in S} v(S)}{\sum_{S \subseteq N} v(S)}=\frac{\psi_{\widetilde{\alpha}^{(B z)}, \lambda=i_{k}}(\overline{\mathrm{Com}}, k \rightarrow j)}{2 \psi_{\alpha, \lambda=+1}(\overline{\mathrm{Com}}, k \rightarrow j)} .
$$

\section{References}

1. M. J. Albizuri and J. Aurrekoetxea. Coalition configurations and the Banzhaf index. Social Choice and Welfare, 26:571-596, 2006.

2. J. Banzhaf. Weighted voting doesn't work: a mathematical analysis. Rutgers Law Review, 19:317-343, 1965.

3. J. S. Coleman. Control of collectivities and the power of a collectivity to act. In B. Lieberman, editor, Social Choice, pages 269-300. New York: Gordon and Breach, 1971.

4. J. S. Coleman. Individual Interests and Collective Action: Selected Essays. Cambridge University Press, 1986.

5. J. Deegan and E. W. Packel. A new index of power for simple n-person games. International Journal of Game Theory, 7:113-123, 1978.

6. P. Dubey and L. S. Shapley. Mathematical properties of the Banzhaf power index. Mathematics of Operations Research, 4:99-131, 1979.

7. D. Felsenthal and M. Machover. The Measurement of Voting Power: Theory and Practice, Problems and Paradoxes. London: Edward Elgar Publishers, 1998.

8. M. Grabisch and M. Roubens. An axiomatic approach to the concept of interaction among players in cooperative games. International Journal of Game Theory, 28:547-565, 1999.

9. M. Grabisch and A. Rusinowska. Measuring influence among players with an ordered set of possible actions. GATE Working Paper, 2008-01, GATE, Ecully, France, 2008.

10. M. Grabisch and A. Rusinowska. A model of influence in a social network. Theory and Decision, 2008. Forthcoming.

11. C. Hoede and R. Bakker. A theory of decisional power. Journal of Mathematical Sociology, 8:309-322, 1982.

12. M. J. Holler and E. W. Packel. Power, luck and the right index. Journal of Economics, 43:21-29, 1983.

13. X. Hu and L. S. Shapley. On authority distributions in organizations: controls. Games and Economic Behavior, 45:153-170, 2003.

14. X. Hu and L. S. Shapley. On authority distributions in organizations: equilibrium. Games and Economic Behavior, 45:132-152, 2003.

15. R. J. Johnston. On the measurement of power: some reactions to Laver. Environment and Planning A, 10:907-914, 1978.

16. T. König and T. Bräuninger. The inclusiveness of European decision rules. Journal of Theoretical Politics, 10:125-142, 1998

17. I. Lindner. The power of a collectivity to act in weighted voting games with many small voters. Social Choice and Welfare, 30:581-601, 2008.

18. G. Owen. Values of games with a priori unions. In Hein and Moeschlin, editors, Essays in Mathematical Economics and Game Theory, pages 77-88. New York: Springer-Verlag, 1977.

19. G. Owen. Modification of the Banzhaf-Coleman index for games with a priori unions. In M. J. Holler, editor, Power, Voting, and Voting Power, pages 232-238. Physica Verlag, Würzburg, 1981.

20. L. S. Penrose. The elementary statistics of majority voting. Journal of the Royal Statistical Society, 109:5357, 1946.

21. D. Rae. Decision-rules and individual values in constitutional choice. American Political Science Review, 63:40-56, 1969.

22. L. S. Shapley. A Boolean model of organization authority based on the theory of simple games. Mimeo, 1994.

23. L. S. Shapley and M. Shubik. A method for evaluating the distribution of power in a committee system. American Political Science Review, 48:787-792, 1954. 